ARTICLE

Received 22 Jul 2016 | Accepted 26 Oct 2016 | Published 4 Jan 2017 DOl: 10.1038/ncomms13816

\title{
PAXX promotes KU accumulation at DNA breaks and is essential for end-joining in XLF-deficient mice
}

Xiangyu Liu', Zhengping Shao ${ }^{1}$, Wenxia Jiang ${ }^{1}$, Brian J. Lee ${ }^{1} \&$ Shan Zha, ${ }^{1,2}$

Non-homologous end-joining (NHEJ) is the most prominent DNA double strand break (DSB) repair pathway in mammalian cells. PAXX is the newest NHEJ factor, which shares structural similarity with known NHEJ factors-XRCC4 and XLF. Here we report that PAXX is dispensable for physiological NHEJ in otherwise wild-type mice. Yet $\mathrm{Paxx}^{-/-}$mice require $\mathrm{XLF}$ and $\mathrm{XIF}^{-/-}$mice require PAXX for end-ligation. As such, $\mathrm{XIF}^{-/}-\mathrm{PaxX}^{-/-}$mice display severe genomic instability and neuronal apoptosis, which eventually lead to embryonic lethality. Despite their structural similarities, only $\mathrm{XIf}^{-/-}$cells, but not $\mathrm{Paxx}^{-/-}$cells require ATM/DNA-PK kinase activity for end-ligation. Mechanistically, PAXX promotes the accumulation of KU at DSBs, while XLF enhances LIG4 recruitment without affecting $\mathrm{KU}$ dynamics at DNA breaks in vivo. Together these findings identify the molecular functions of PAXX in KU accumulation at DNA ends and reveal distinct, yet critically complementary functions of PAXX and XLF during NHEJ.

\footnotetext{
${ }^{1}$ Department of Pathology and Cell Biology, College of Physicians and Surgeons, Institute for Cancer Genetics, Columbia University, 1130 Saint Nicholas Avenue, Room 501, New York City, New York 10032, USA. ${ }^{2}$ Division of Pediatric Oncology, Hematology and Stem Cell Transplantation, Department of Pediatrics, College of Physicians \& Surgeons, Columbia University, 1130 Saint Nicholas Avenue, Room 501, New York City, New York 10032, USA.

Correspondence and requests for materials should be addressed to S.Z. (email: sz2296@cumc.columbia.edu).
} 
D NA double strand breaks (DSBs) are the most severe form of DNA damages. In mammalian cells, most DSBs are repaired by the non-homologous end-joining (NHEJ) pathway, which directly ligates two DNA ends together. NHEJ is not only critical for general DNA repair, but also exclusively required for lymphocyte development and the viability of post-mitotic neurons ${ }^{1}$. Accordingly, human patients and mouse models with defects in any of the seven well-characterized NHEJ factors develop severe primary immunodeficiency and variable degrees of microcephaly ${ }^{1,2}$. Among these factors, KU70 and KU80 (KU86 in human) form a heterodimer (referred to as KU) that binds and protects DNA ends from nucleases and recruits the DNA-dependent protein kinase catalytic subunit (DNA-PKcs). DNA-PKcs in turn recruits and activates the Artemis endonuclease for end-processing (for example, hairpin opening). Finally, DNA Ligase 4 (Lig4), in complex with XRCC4 and XLF (also called Cernunnos or NHEJ1), ligates the ends together. The five 'core' NHEJ factors, KU70, KU80, XRCC4, Lig4 and XLF, are essential for end-ligation and conserved in all eukaryotes. In contrast, complete loss of DNA-PKcs or Artemis, both of which are only found in vertebrates, abolishes end-processing (that is, hairpin opening), but not end-ligation. We recently reported that expression of a kinase-dead DNA-PKcs protein blocks end-ligation, suggesting that DNA-PKcs coordinates end-processing and end-ligation during NHEJ through auto-phosphorylation ${ }^{3}$.

Normal lymphocyte development requires the assembly of functional antigen receptor genes from germline variable (V), diversity (D) and joining (J) gene segments through $\mathrm{V}(\mathrm{D}) \mathrm{J}$ recombination. $\mathrm{V}(\mathrm{D}) \mathrm{J}$ recombination is initiated by products of the recombination activating gene (RAG), which cleaves germline antigen receptor genes to generate a pair of blunt signalling ends (SEs) and a pair of hairpin coding ends $(\mathrm{CEs})^{4}$. The two SEs are precisely joined by the core NHEJ factors to form a signalling joint (SJ), while the two hairpin CEs are first opened by DNA-PKcs and Artemis before being joined by the core NHEJ factors to generate a coding joint (CJ). The unique structures of CEs and SEs and the exclusive requirement for NHEJ during $\mathrm{V}(\mathrm{D}) \mathrm{J}$ recombination provide a sensitive and well-characterized physiological system to evaluate end-ligation and end-processing functions of the NHEJ factors. While loss of the conserved core NHEJ factor abrogates both CJs and SJs, complete loss of DNA-PKcs or Artemis only abrogates CJ formation. CJs comprise part of the exons encoding the antigen receptor genes and are thus required for lymphocyte development. As such, loss of all previously characterized NHEJ factors, except XLF (see below), abrogates lymphocyte development at the progenitor stage, leading to $\mathrm{T}^{-} \mathrm{B}^{-}$severe combined immunodeficiency (SCID) in patients and animal models. RAG holds the DNA ends generated during $\mathrm{V}(\mathrm{D}) \mathrm{J}$ recombination in a post-synaptic complex, which promotes end-ligation in the absence of $\mathrm{XLF}^{5}$. In peripheral lymphoid organs (for example, the spleen), naive B cells further modify the constant region of the immunoglobulin heavy chain (IgH) through class switch recombination (CSR) to achieve different antibody effector functions. CSR is initiated by activation-induced deaminase. The DSB intermediates generated during CSR, which do not harbour hairpins, are joined together primarily by core NHEJ factors to complete CSR. Thus, loss of any core NHEJ factor, including XLF, leads to frequent $I g H$ chromosomal breaks and reduces CSR efficiency by $50-75 \%$ (refs 6-8). In addition, post-mitotic neurons require NHEJ for survival. Complete loss of core NHEJ factors causes widespread neuronal apoptosis and eventually late embryonic lethality in Lig4or XRCC4-deficient mice $1,3,9-11$. Efficient and accurate NHEJ on chromatinized DNA also requires ataxia-telangiectasia mutated (ATM) kinase and ATM-mediated DNA damage responses ${ }^{6}$. While not essential for $\mathrm{V}(\mathrm{D}) \mathrm{J}$ recombination, loss of ATM or its substrates (for example, H2AX and 53BP1) abrogates chromosomal $\mathrm{V}(\mathrm{D}) \mathrm{J}$ recombination in XLF-deficient mice and cells, underscoring the intricate interaction between DNA damage response and core NHEJ factors ${ }^{12-15}$.

Paralog of XRCC4 and XLF (PAXX, also called C9ORF142 or XLS) was proposed as a NHEJ factor based on its structural similarity with XRCC4 and XLF ${ }^{16-18}$. Since patients or animal models with defects in PAXX are not yet found, the physiological function of PAXX remains largely unknown. XRCC4, XLF and PAXX all have an N-terminal globular head domain followed by a C-terminal coil-coiled stalk, and each forms stable homodimers via their respective coil-coiled stalks ${ }^{19,20}$. XRCC4 deficiency phenocopies Lig4 deficiency, likely because the stalk of the XRCC4 homodimer binds and stabilizes Lig4 protein. In contrast, the coiled-coil stalks of XLF and PAXX are much shorter and do not bind Lig4 directly ${ }^{16,17,21,22}$. While not absolutely required for NHEJ, XLF dimers promote end-ligation by forming high-order helical filaments with XRCC4 dimers through direct interactions between their respective head domains ${ }^{7,12,23,24}$. PAXX does not directly interact with either XLF or XRCC4. Instead, PAXX binds $\mathrm{KU}$ through a conserved C-terminal region ${ }^{16-18}$. A PAXX mutant that cannot bind to $\mathrm{KU}$ fails to rescue the severe IR sensitivity in human cells ${ }^{16,18}$. Notably, co-deletion of PAXX partially rescues the severe IR sensitivity of XRCC4-knockout DT40 cells ${ }^{17}$, but accentuates the zeocin sensitivity of XLF-deficient HCT116 cells ${ }^{24}$. The exact role of PAXX in NHEJ and DSB repair is yet to be demonstrated.

To elucidate the functions of PAXX in NHEJ and determine the physiological function of PAXX in vivo, we generated PAXX-deficient mice. Interestingly, although not required for NHEJ in otherwise wild-type mice, PAXX is essential for both chromosomal and extra-chromosomal end-ligation in Xlf-deficient animals. Our data further suggest that PAXX and XLF support NHEJ through distinct mechanisms, with PAXX facilitating the accumulation of $\mathrm{KU}$ at DNA ends and XLF enhancing the recruitment of Lig4 to achieve efficient end-ligation together.

\section{Results}

Generation of PAXX-deficient mice. To determine the physiological functions of PAXX, we generated Paxx knockout mice $\left(\right.$ Pax $\left.^{-I^{-}}\right)$by a gene targeting strategy in which all coding exons of the Paxx gene and part of the non-coding exon 1 were replaced by a Neomycin resistant (NeoR) cassette flanked by frt sequences (Fig. 1a). Correct targeting, which removes an EcoRV site within the Paxx gene, was confirmed by Southern blotting analyses (Fig. 1b). Eight independently targeted embryonic stem (ES) cell clones (in 129/sv background) were obtained and two were injected for germline transmission. The resulting chimeras were bred with ROSA26 ${ }^{\text {FLIP/FLIP }}$ mice expressing FLIPase constitutively $^{25}$ (Jackson Laboratory, Stock No. 003946) to remove the NeoR cassette and generate $P_{a x x}+1-$ mice. Intercrosses between Paxx $^{+/-}$mice produced Paxx $^{-/}$pups at the expected Mendelian ratio (Fig. 1c). Southern blotting confirmed complete deletion of the Paxx gene in $\mathrm{Paxx}^{-1-}$ mice and western blotting validated the absence of PAXX protein in Paxx ${ }^{-1}-$ murine embryonic fibroblasts (MEFs) (Fig. 1d,e). Both male and female $P a x x^{-1-}$ mice are of normal size (Fig. 1f), and spontaneous tumour development has not yet been observed. Given the embryonic lethality and severe growth retardation of XRCC4- and KU-deficient mice, these data suggest that PAXX is not essential for end-ligation in mice. 


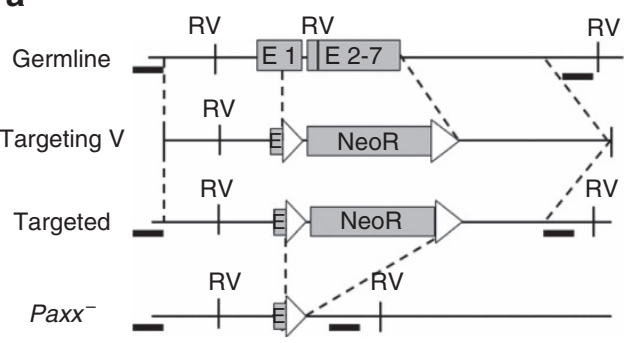

b

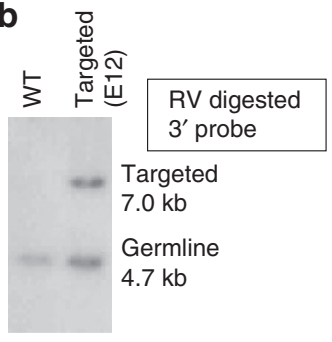

C

\begin{tabular}{cccccc}
\hline Paxx & $+/+$ & $+/-$ & $-/-$ & Total & $P$ value \\
\hline Birth & 2 & 7 & 5 & 14 & 0.53 \\
\hline
\end{tabular}

d

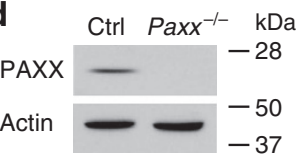

e

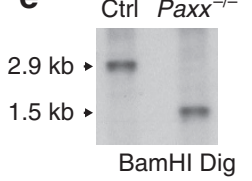

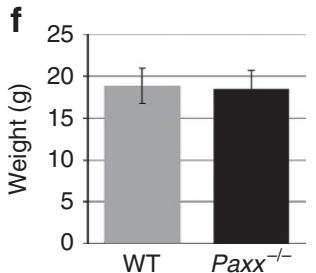

Figure 1 | Generation of $\mathbf{P a x x}{ }^{-/-}$mice. (a) The schematic diagram represents the murine Paxx locus (top), targeting vector (2nd row), targeted allele (3rd row) and the neo-deleted allele (Paxx ${ }^{-}$, bottom). The $5^{\prime}$ and $3^{\prime}$ probe are marked as thick black lines. The exons and frt sites are shown as solid boxes and open triangles, respectively. Restriction site designation: RV = EcoRV. The map is not drawn to scale. (b) Southern blot analyses of EcoRV-digested DNA from WT and Paxx +/Targeted ES cells, blotted with the $3^{\prime}$ probe. (c) The number of live-birth mice obtained from intercrossing Paxx $+/-$ mice. The $P$ value was calculated with the chi-square test. (d) Western blot for PAXX in primary murine embryonic fibroblasts derived from E14.5 WT or Paxx $-/-$ embryos. (e) Southern blot analyses of BamHI-digested DNA from Paxx $+/+$ and Paxx ${ }^{-/}-$mice tissue DNA and probed with mPaxx probe diagrammed in Fig. 5a. (f) The total body weight of $\mathrm{Paxx}^{+/+}$and Paxx ${ }^{-/-}$littermates at $\sim 50$ days of age. The data represent the average and s.d. of more than four mice of each genotype.

Lymphocyte development is normal in $\operatorname{Paxx}^{-1-}$ mice. Since $X^{-I}-$ mice develop normally, but have reduced lymphocyte cellularity and CSR defects ${ }^{7}$, we next analysed $\mathrm{Paxx}^{-I}$ - mice for lymphocyte development. The weights and total cellularity of the thymus and spleens of 7-8-week-old $P_{a x x}-1-$ mice (4 male, 6 female) were indistinguishable from those of Paxx $+1+(4$ male, 3 female) littermates (Fig. 2a and Supplementary Fig. 1A). Fluorescence activated cell sorting (FACS) analyses showed that the frequencies of immature pro- $\mathrm{B}\left(\mathrm{CD} 43^{+} \mathrm{B} 220^{+} \mathrm{IgM}^{-}\right)$, pre- $\mathrm{B}$ $\left(\mathrm{CD}_{3}{ }^{-} \mathrm{B}_{220}{ }^{+} \mathrm{IgM}^{-}\right)$, newly generated naive $\mathrm{B}\left(\mathrm{IgM}^{+} \mathrm{B} 220^{\text {low }}\right)$ and re-circulating $\mathrm{B}\left(\mathrm{IgM}^{+} \mathrm{B} 220^{\mathrm{hi}}\right)$ cells in bone marrow from $\mathrm{Paxx}^{-1-}$ mice were also comparable to those of $\mathrm{Paxx}{ }^{+/+}$mice (Fig. $2 \mathrm{~b}$ and Supplementary Fig. 1C). Successful V(D)J recombination at the $\mathrm{IgH}$ locus is required for the transition from pro- $\mathrm{B}$ to pre- $\mathrm{B}$ cells. The ratio of bone marrow-derived pre$\mathrm{B} /$ pro-B cells was the same in both Paxx $^{-1-}$ mice and their Paxx ${ }^{+/+}$littermates (Fig. 2b). Likewise, the number and frequency of $\mathrm{T}$-cell progenitors and mature $\mathrm{T}$ cells are also indistinguishable in Paxx $-1-$ and Paxx ${ }^{+/+}$mice (Fig. $2 \mathrm{c}$ and Supplementary Fig. 1B). Sequential rearrangements of the TCR $\alpha$ locus in $\mathrm{CD} 4+\mathrm{CD} 8+$ double positive (DP) thymocytes are coupled with both positive and negative selections, creating a stressful situation that reveals minor $\mathrm{V}(\mathrm{D}) \mathrm{J}$ recombination defects in ATM or 53BP1-deficient cells previously, indicated by reduced surface expression of TCR $\beta$ and its co-receptor CD3 in the DP cells $^{26,27}$ (Supplementary Fig. 1D). Nevertheless, surface expression of TCR $\beta / C D 3$ in DP cells was not affected by PAXX deficiency (Fig. 2c). Consistent with normal lymphocyte development, endogenous $\mathrm{V}(\mathrm{D}) \mathrm{J}$ recombination junctions in $\mathrm{Paxx}^{-1-}$ and $\mathrm{Paxx}^{+/+}$mice are also indistinguishable (Supplementary Table 1). Moreover, CSR is not affected by Paxx deficiency. In vitro stimulation with bacterial lipopolysaccharide (LPS) and interleukin 4 (IL-4) induced robust CSR to IgG1, and expression of surface IgG1 in $\sim 30 \%$ of Paxx ${ }^{+/+}$as well as Paxx ${ }^{-I-}$ B cells in 4 days (Figs 2 d,e and
Supplementary Fig. 2A), while only $\sim 10 \%$ of $\mathrm{Xlf}^{-/}-$B cells express IgG1 at Day 4, consistent with 50-75\% reduction in $\mathrm{CSR}^{7}$. Likewise, proliferation of in vitro-stimulated $\operatorname{Paxx}^{-1-}$ and Paxx $+1+$ B cells is also similar (Supplementary Fig. 2B). Together, these results indicate that PAXX, unlike XLF and other NHEJ factors, is not required for either $V(D) J$ recombination or CSR, two physiological gene rearrangements mediated by NHEJ.

$X f^{-/-}$Paxx $^{-/-}$mice die during embryonic development. Previous studies by us and others suggest that the apparently efficient chromosomal end-joining in XLF-deficient cells is quite vulnerable and requires both ATM and DNA-PK kinase activities and their substrates (for example, 53BP1 and $\mathrm{H} 2 \mathrm{AX})^{5,12-14,24,28}$. Given the structural similarity between PAXX and XLF, we asked whether PAXX is necessary for end-joining in $X l f^{-1-}$ mice. Intercrossing double-heterozygous

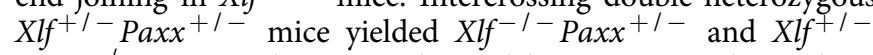
$P a x x^{-1-}$ pups at the expected Mendelian ratios, similar to those of $X l f^{-1-} \mathrm{Paxx}^{+/+}$and $X l f^{+/+} \mathrm{Paxx}^{-1-}$ pups (Fig. 3a). However, double-homozygous $X l f^{-1-} \operatorname{Paxx}^{-1}-$ progeny were not obtained, suggesting embryonic lethality (Fig. 3a). Embryonic lethality caused by Lig4 or Xrcc4 deficiency occurs shortly after embryonic day 15.5 (E15.5) with severe neuronal apoptosis ${ }^{3,9-11}$. Notably at E14.5, $\mathrm{Xlf}^{-/}-\mathrm{Paxx}^{-/-}$embryos, which were slightly smaller in size than their littermates, were found at the expected Mendelian ratios (Fig. 3b). Neuronal apoptosis, as indicated by condensed nuclei and positive staining for cleaved (activated) Caspase 3, was rare in $X l f^{-/-}$or $P a x x^{-/-}$embryos and increased $>40$-fold in $X l f^{-1-} \mathrm{Paxx}^{-1-}$ embryonic brains, to a level comparable to end-ligation defective $X r c c 4^{-/-}$ or DNA-PKcs KD/KD embryos (Fig. 3c,d) ${ }^{3,11}$. Moreover, apoptotic inclusions were most prominent in the post-mitotic intermediate zone, but not the proliferating ventricular zone, 


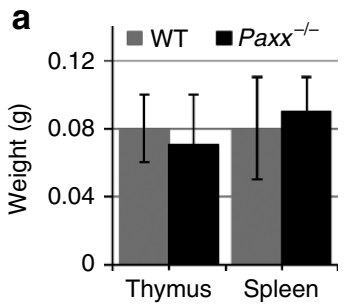

b
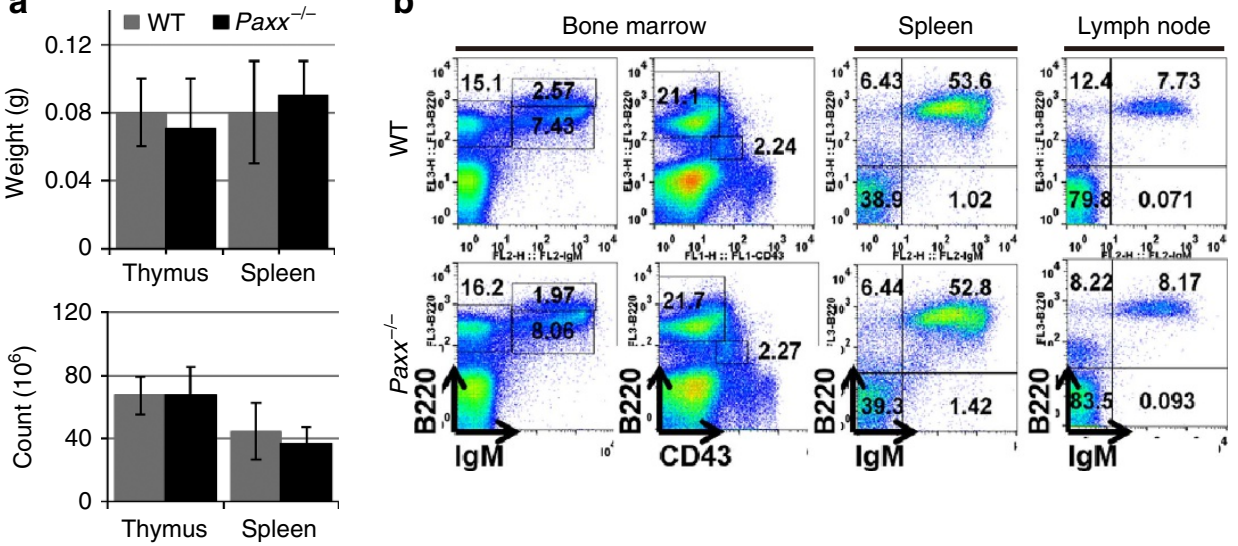

C
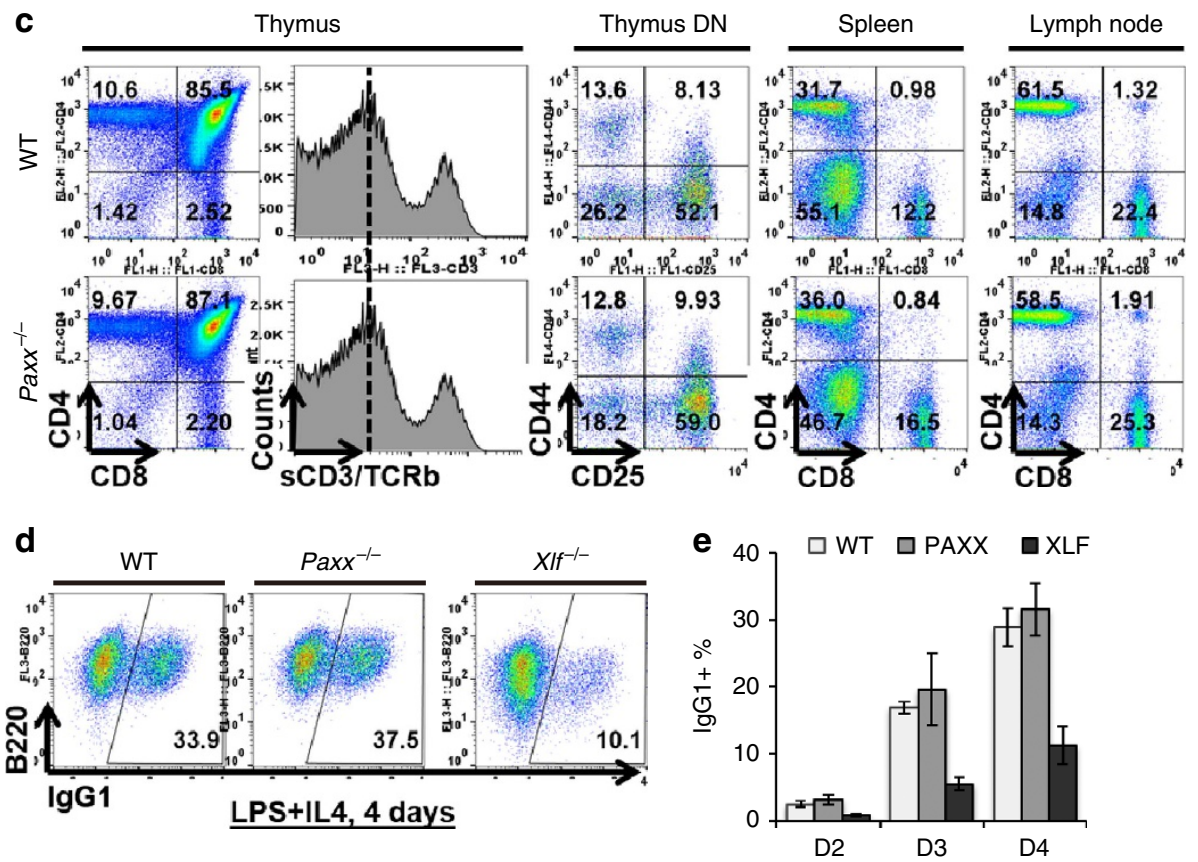

Figure 2 | Lymphocyte development in Paxx ${ }^{-/-}$mice. (a) Weight and total cell count of thymus and spleen from Paxx $+/+$and Paxx $-/-$littermates at $\sim 50$ days of age (7-8 weeks). The data represent the average and s.d. of $>3$ mice of each genotype. (b,c) Representative flow cytometry analyses of 7-8-week-old Paxx $+/+$ and Paxx ${ }^{-/-}$mice. (d) Representative flow cytometry analysis of IL-4/LPS stimulated B cells (CD43- splenocytes) from $\mathrm{Paxx}^{+/+}, \mathrm{Paxx}^{-/-}$and $\mathrm{XIF}^{-/-}$mice. (e) Statistical analyses of the class-switching results in $\mathbf{d}$. Three biological repeats were performed for each genotype. The bar graphs represent the average and s.e.

of $X l f^{-1-} \mathrm{Paxx}^{-/-}$embryos, in a manner reminiscent of $X r c c 4^{-1-}$ embryos $^{29}$. Together, these findings reveal a critical role of PAXX in the survival of post-mitotic neurons and embryonic development in mice lacking XLF.

Severe genomic instability in $\boldsymbol{X l f}^{-/-} \boldsymbol{P a x x}^{-/-}$cells. To determine whether DNA repair defects and genomic instability contribute to the embryonic lethality of Xlf/Paxx double-deficient mice, we generated MEFs from E14.5 Xlf ${ }^{-/-}$Paxx $^{-/-}$and control (wild type (WT) and Xlf- or Paxx single deficient) embryos. Consistent with our previous study ${ }^{30}$ and the normal development of $\mathrm{Paxx}^{-1-}$ mice, the $\mathrm{Paxx}^{-1-}$ and $\mathrm{Xlf}^{-1-}$ primary MEFs proliferated at rates comparable to that of WT MEFs. In contrast, $X l f^{-1-}$ Paxx $^{-1-}$ MEFs displayed severe proliferation defects and failed to thrive at early passages (Fig. 4a). Correspondingly, cell cycle analyses revealed $\sim 25 \%$ reduction (from 25 to $\sim 18 \%$ ) in the percentage of $\mathrm{S}$ phase $(\mathrm{BrdU}+$ ) cells from $X l f^{-1-}$ Paxx ${ }^{-1-}$ primary MEFs (Supplementary Fig. 3A). Despite normal proliferation, $\operatorname{Paxx}^{-1-}$ MEFs were moderately, yet significantly, more sensitive to IR and Etoposide than Paxx ${ }^{+/+}$cells, while less sensitive than $X l f^{-/-}$MEFs (Fig. 4b and Supplementary Fig. 3B). Meanwhile, $X l f^{-/-}$Paxx $^{-1-}$ MEFs were highly sensitive to IR and Etoposide, consistent with their severe proliferation defects (Fig. 4b). In addition, Paxx ${ }^{-1-}$ and $X l f^{-1-}$ primary MEFs are very moderately sensitive to hydroxyurea (HU) at the highest dose, while $X l f^{-/-}$ Paxx $^{-1-}$ primary MEFs are not apparently sensitive to HU (Supplementary Fig. 3C). Since HU primarily targets replicating cells, the lack of HU sensitivity might be due to the lower frequency of $S$ phase cells among $X^{-1} f^{-1}$ Paxx $^{-1}$ primary MEFs (Supplementary Fig. 3A). Cytogenetic analyses of spontaneous genomic instability using telomere-specific fluorescence in situ hybridization ( $\mathrm{T}$ FISH) revealed that $18.9 \pm 2.6 \% X^{-1} f^{-} \mathrm{Paxx}^{-/}$primary MEFs had at least one cytogenetic abnormality, in comparison with 
a

\begin{tabular}{ccccc}
\multicolumn{2}{l}{$\mathrm{XIf}^{+/-}$or $\mathrm{XIf}^{+/+}$} \\
\hline Paxx & $+/+$ or $+/-$ & $-/-$ & Total & $P$ value \\
\hline E14.5 & 8 & 5 & 13 & $>0.5$ \\
\hline Birth & 14 & 6 & 20 & $>0.5$ \\
\hline XIf & & & & \\
\hline Paxx & $+/+$ or $+/-$ & $-/-$ & Total & $P$ value \\
\hline E14.5 & 5 & 3 & 8 & $>0.5$ \\
\hline Birth & 17 & 0 & 17 & $<0.05$ \\
\hline
\end{tabular}

C
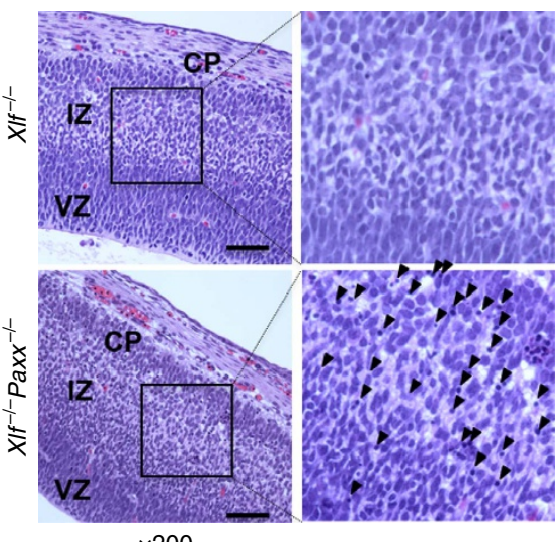

$\times 200$ b

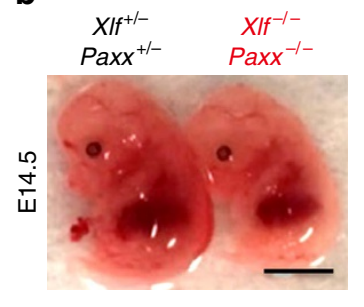

d

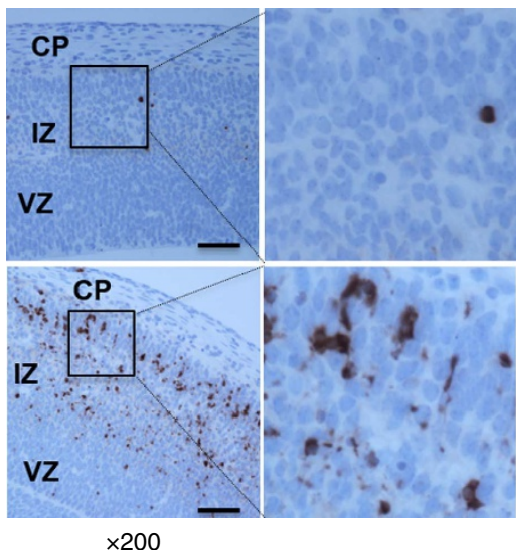

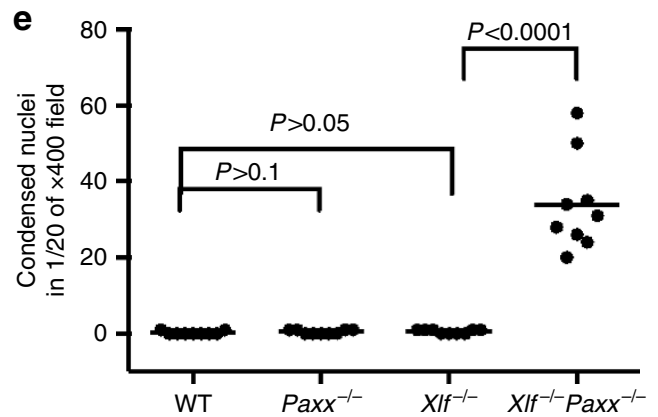

Figure 3 | Embryonic lethality and severe neuronal apoptosis in $\mathbf{X I f}^{-\boldsymbol{I}^{-}}$Paxx $^{-1}$ - mice. (a) Live birth and E14.5 embryos obtained from intercross between XIf and Paxx-deficient mice. (Upper table) All E14.5 embryo or birth with $\mathrm{XIf}^{+/}+$or $X I f^{+/-}$genotypes and various Paxx genotypes. (Lower table) All E14.5 embryos or birth with XIf ${ }^{-/-}$genotypes. XIf ${ }^{-/-P a x X^{-/}-}$were not found at birth and found at expected ratio at E14.5 (P>0.5). The $P$ value was calculated with the chi-square test. (b) Representative $X_{I f}{ }^{+/-}$Paxx ${ }^{+/-}$and littermate $X^{-1}{ }^{-/-}$Paxx ${ }^{-/-}$embryos at E14.5. The length of the scale bar is $1 \mathrm{~cm}$. (c) Haematoxylin and eosin (H\&E) staining of E14.5 embryonic brains from littermate XIf- ${ }^{-}-$or XIf ${ }^{-/-P a x x}{ }^{-/-}$mice. The ventricular zone (VZ) contains the proliferating cells. The intermediate zone (IZ) and cortical plate (CP) contain post-mitotic neurons. Black arrowheads denote condensed nuclei. The scale bar in c stands for $100 \mu \mathrm{m}$. (d) Immunostaining of E14.5 embryonic brain using antibodies against Cleaved (activated) Caspase3. Brown staining represents apoptotic cells. The scale bar in $\mathbf{d}$ stands for $100 \mu \mathrm{m}$. (e) The frequency of condensed nuclei per 1/20 of $\times 400$ field in E14.5 brain were quantified. The data represent nine different fields from at least two biological repeats of each genotype. The $P$ value was calculated with the chi-square test.

only $2.0 \pm 0.1 \%$ in $X l f^{+/+} \operatorname{Paxx}^{+/+}(W T), \quad 3.4 \pm 0.3 \%$ in $\mathrm{Paxx}^{-1-}$ and $9.6 \pm 0.1 \%$ in $\mathrm{Xlf}^{-1-}$ primary MEFs (Fig. $4 \mathrm{c}$ and Supplementary Fig. 3D). T-FISH can distinguish two types of cytogenetic abnormalities ${ }^{31}$ : (1) chromosomal breaks involving both sister chromatids, which reflect repair defects occurring in the G1 phase of the cell cycle, and (2) chromatid breaks involving only one chromatid, which reflect damage that occurs after DNA replication. Of note, almost all of the cytogenetic abnormalities in $\mathrm{Xlf}^{-/-} \mathrm{Paxx}^{-/}$cells were chromosomal breaks (Fig. 4c and Supplementary Fig. 3D), similar to the cytogenetic defects observed in cells deficient for other core NHEJ factors. Together, these findings reveal a critical complementary function between PAXX and XLF in maintaining genomic stability during normal cell growth and in response to IR or etoposide challenges. The skew towards chromosomal breaks together with the presense of IR and etoposide hypersensitivity strongly suggest defects in the NHEJ pathway in $X l f^{-I-} \mathrm{Paxx}^{-/-}$cells.

End-joining is abrogated in $X l f^{-/-} \operatorname{Paxx}^{-/-}$cells. To further characterize the function of PAXX and XLF in NHEJ, we used CRISPR/Cas9 technology to delete the entire murine Paxx gene from WT or $X l f^{-/-} E \mu-B c l 2^{+}$Abelson Murine Leukemia Virus (A-MuLV)-transformed pre-B cell lines ( $\mathrm{v}-\mathrm{abl}$ cells) (Fig. 5a). These CRISPR/Cas9-deleted cells are referred to as $\mathrm{Paxx}^{\mathrm{S} / \Delta}$, to distinguish them from germline $\mathrm{Paxx}^{-/-}$mice and cells. Successful deletion of the Paxx gene was monitored by Southern blotting (Fig. 5b) and the absence of PAXX protein was confirmed by western blot analyses (Fig. 5c). 
a

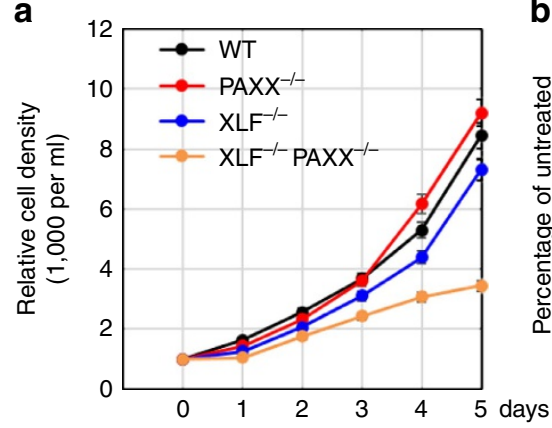

b

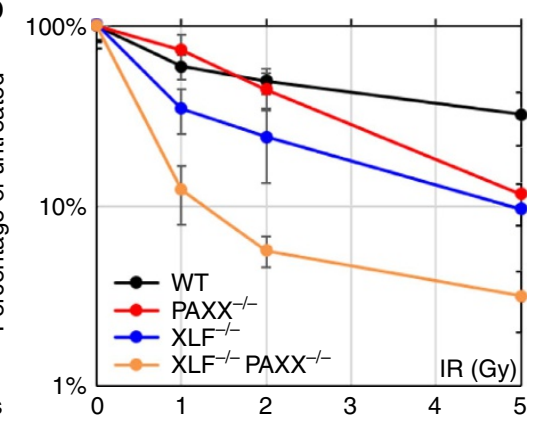

C

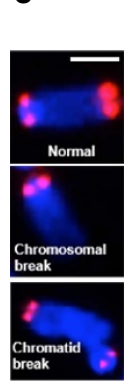

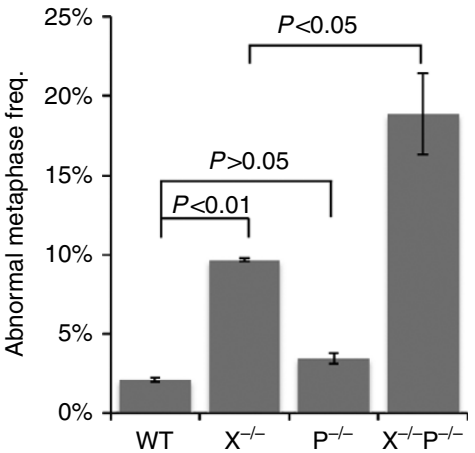

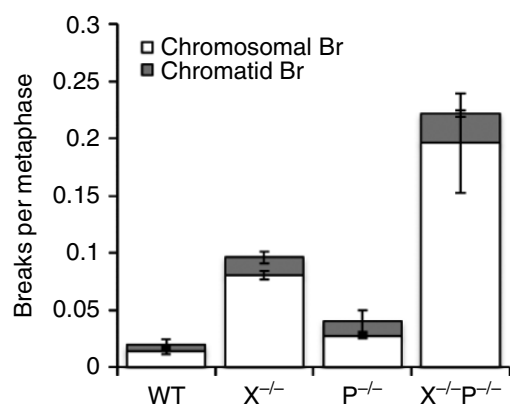

Figure 4 | Proliferation defects and IR hypersensitivity of $\mathbf{X I f}^{- \text {/ }}-$ Paxx $^{- \text {/ }}$ - MEFs. (a,b) Proliferation and IR sensitivity assays were performed using P2 primary MEFs with different genotypes. Three independent repeats were performed. The average and s.d. were plotted. The $Y$ axis is the relative cell number measured by hemocytometer (for $\mathbf{a}$ ) or the fluorescence-based nucleotide dye (CyQuant Cell Proliferation Assay, Invitrogen, CA) (for b).

(c) Left: the examples of normal chromosome, chromosomal breaks and chromatid breaks (one of two sister chromatids is broken). Middle: frequency of metaphases with one or more cytogenetic abnormalities in P1 primary MEFs of different genotypes. At least two independently derived lines were assayed for each genotype. The scale bar stands for $1 \mu \mathrm{m}$. Right: the frequency of chromatid (grey box) and chromosomal (white box) breaks measured by T-FISH analyses in P1 primary MEF of different genotypes. The average and s.d. of at least three independent biological repeats were shown. $X^{-/-}=X^{-} f^{-/-}$, $\mathrm{P}^{-/-}=\mathrm{Paxx}^{-/-}$and $\mathrm{X}^{-/-} \mathrm{P}^{-/-}=\mathrm{XIf}^{-/-} \mathrm{Paxx}^{-/-}$. The $P$ value was calculated by two-tailed Student's $t$-test. The raw data were summarized in Supplementary Fig. 3D.

Consistent with the results from primary MEFs (Fig. 4b), v-abl kinase-transformed $X l f^{-I}-\mathrm{Paxx}^{\Delta / \Delta}$ pre-B cells were hypersensitive to IR, similar to the $X r c c 4^{-/}$control, while $P a x x^{\triangle /}$ pre-B cells are, at most, moderately sensitive to IR (Fig. 5d). Also consistent with the data from MEFs, Xlf $f^{-1}-\mathrm{Paxx}^{\Delta / \Delta}$ pre-B cells display higher levels of spontaneous genomic instability, especially chromosomal breaks (Fig. 5e and Supplementary Fig. 3E).

To measure NHEJ function specifically, we utilized the chromosomal $V(D) J$ recombination assay ${ }^{15}$. In this system, v-abl kinase inhibitor STI571 (also called Gleevec) induces a G1 phase cell cycle arrest in the $\mathrm{v}$-abl cells that allows accumulation of RAG protein, which initiates efficient $V(D) J$ recombination at a chromosomal integrated pMX-INV inversional substrate ${ }^{15}$. pMX-INV contains an inverted green fluorescent protein (GFP) cassette flanked by recombination signal sequences (RSS, triangles in Fig. 6a) that can be cleaved and inverted back to the same orientation as the promoter by RAG-mediated recombination reaction. NHEJ-mediated repair and the formation of both SJs and CJs are necessary and sufficient for the expression of GFP protein (Fig. 6a) ${ }^{3,15}$. Using this assay, we found that the integrated pMX-INV substrate was rearranged robustly in two isogenic clones of $\operatorname{Paxx}^{\Delta / \Delta}$ cells derived from parental WTInv4 cells (pMX-INV at the same genomic locus in both WT and $\mathrm{Paxx}^{\mathrm{A} / \mathrm{J}}$ cells), as measured by the appearance of CJs in Southern blots (Fig. 6b and Supplementary Fig. 4B) and the accumulation of GFP expressing cells by FACS (Supplementary Fig. 4A). Thus, PAXX itself is not required for chromosomal $\mathrm{V}(\mathrm{D}) \mathrm{J}$ recombination. In contrast, while parental $X l f^{-1-}$ cells can be induced to express GFP efficiently, $X l f^{-1-} \mathrm{Paxx}^{\Delta / \Delta}$ cells failed to express GFP upon STI571 induction and accumulated both $3^{\prime}$-CEs and the small CEs-SEs fragment similar to those seen in $\mathrm{Xrcc4}^{-1-}$ cells, indicative of end-ligation defects (Supplementary Fig. 4A and Fig. 6c). Ectopic expression of either PAXX or XLF protein in $\mathrm{Xlf}^{-{ }^{-}-} \mathrm{Paxx}^{\Delta / \Delta}$ B cells restored $\mathrm{V}(\mathrm{D}) \mathrm{J}$ recombination, supporting the specificity of Paxx deletion by CRISPR/Cas9 (Fig. 6d, Supplementary Fig. 4C). Moreover, Xlf ${ }^{-1-} \mathrm{Paxx}^{\Delta / \Delta}$ B cells, similar to $X r c c 4^{-/-}$cells, also failed to repair chromosomal DSBs generated by Ppol endonuclease, indicating that their end-ligation defects are not limited to $V(D) J$ recombination (Fig. 6e). These findings, together with the severe neuronal apoptosis of $\mathrm{Xlf}^{-/-} \mathrm{Paxx}^{-/-}$mice, reveal a critical function for PAXX in NHEJ-mediated end-ligation that is otherwise masked by XLF.

PAXX and XLF have distinct functions during NHEJ. Given their structural similarities, it is conceivable that PAXX and XLF are functionally equivalent. However, ATM (KU55933) or DNA-PK (NU7441) kinase inhibitors did not block the formation of CJ products or the expression of GFP in Paxx $^{-1-}$ cells, despite the fact that $X l f^{-1-}$ pre-B cells require both ATM and DNA-PK kinase activity for efficient $V(D) J$ recombination (Figs $6 \mathrm{~b}$ and $7 \mathrm{a}$, and Supplementary Fig. $4 \mathrm{~A})^{12,28}$. Moreover, overexpression of PAXX was not sufficient to rescue the 
a

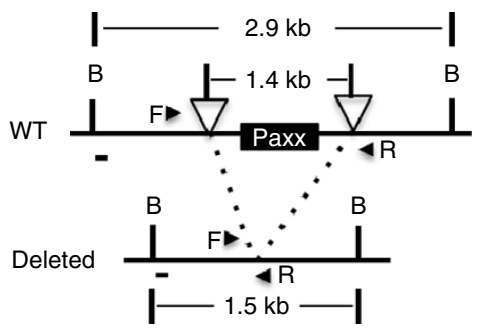

C

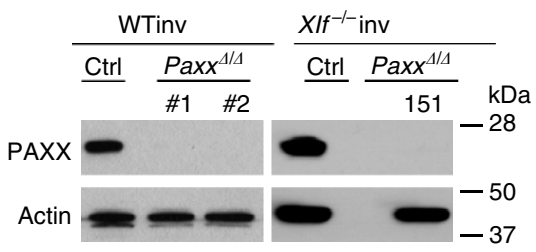

b
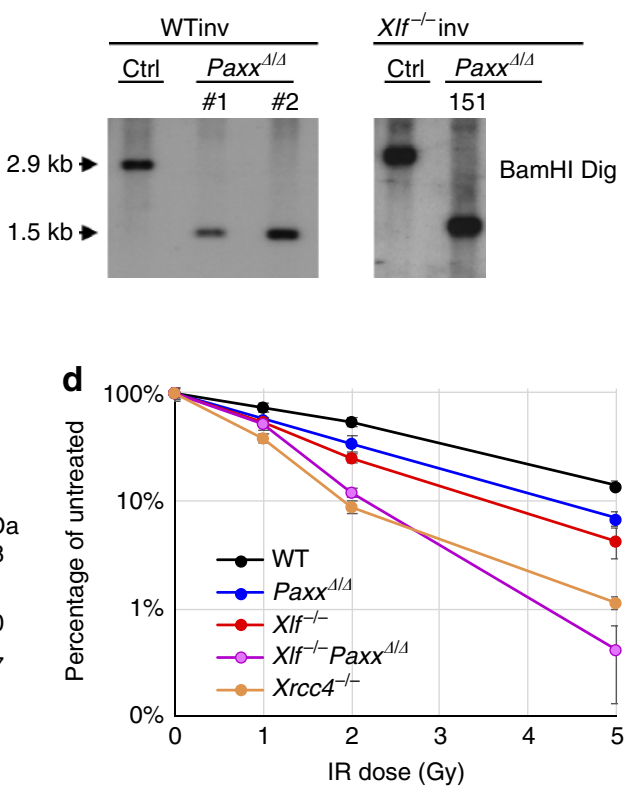

e

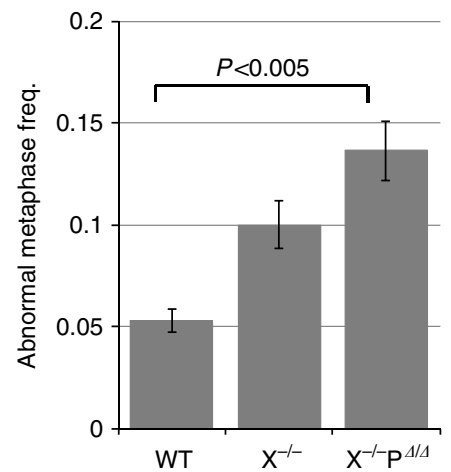

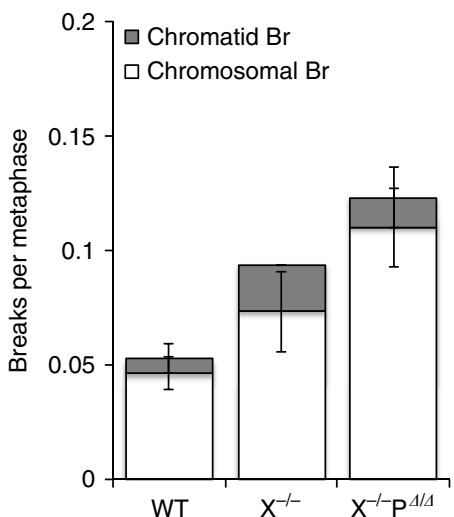

Figure 5 | Generation and characterization of isogenic $\mathbf{X I f}{ }^{-/}-$Paxx $^{\Delta / \Delta}$ pre-B cell lines. (a) Scheme for murine Paxx locus and CRISPR/Cas 9 mediated knockout strategy. The open triangles indicate the location of the guided RNA. The horizontal arrows indicate the location of the primers used for primary screen. Southern blot confirmation was performed using BamHI-digested DNA with the mPaxx probe (show as thick black line). (b,c) Southern blot and

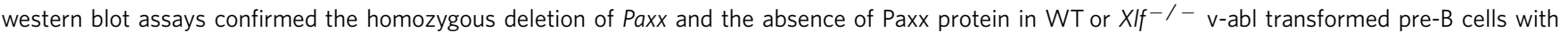
inverted substrates. (d) IR sensitivity of WT, Paxx ${ }^{-/-}, X^{-1} f^{-}, X I f^{-/-}$Paxx ${ }^{4 / \Delta}$ and $X_{r c c} 4^{-/-}$pre-B cells measured, determined by cell counting. At least triplicates were plated and counted for each genotype at each dosage. (e) Left: frequency of metaphases with cytogenetic abnormalities of WT, $\mathrm{XIf}^{-/-}$or XIf ${ }^{-/}-\mathrm{Paxx}^{\Delta / \Delta} \mathrm{v}$-abl transformed pre-B cells. At least three independent experiments were performed. The data represent the average and the s.d. of the three repeats. Right: the frequency of chromatid (grey box) and chromosomal (white box) breaks measured by T-FISH analyses in the pre-B cells. The raw data were summarized in Supplementary Fig. $3 \mathrm{E} . \mathrm{X}^{-/-}=\mathrm{XIf}^{-/-}, \mathrm{P}^{\Delta / \Delta}=\mathrm{Paxx}^{\Delta / \Delta}$ and $\mathrm{X}^{-/-} \mathrm{P}^{\Delta / \Delta}=\mathrm{XIf}^{-/-} \mathrm{Paxx}^{\Delta / \Delta}$.

end-ligation defects caused by ATM inhibition in Xlf-deficient cells (Supplementary Fig. 4A). Together these findings suggest that PAXX cannot replace XLF, and XLF and PAXX likely have distinct functions during NHEJ.

Given $X l f^{-1-}$ cells require ATM as well as PAXX for end-ligation, we asked whether PAXX affects ATM activation. IR- or camptothecin (CPT)-induced phosphorylation of specific ATM substrate KAP1 was unaffected in Paxx ${ }^{-/}$cells (Fig. 7b and Supplementary Fig. 5B). Moreover, while ATM is required for chromosomal end-ligation and dispensable for end-ligation of extra-chromosomal (episomal) DNA in XLF-deficient cells ${ }^{12}$, both chromosomal and extrachromosomal SJs formation decreased nearly 100-fold in $X l f^{-1-} \mathrm{Paxx}^{\Delta / \Delta}$ cells, in comparison with WT or $X l f^{-1-}$ or $\mathrm{Paxx}^{\Delta / \Delta}$ single deficient cells (Fig. $7 \mathrm{c}$ and Supplementary Fig. 5A). Together, these results suggest a direct function of
PAXX in DNA end-ligation that is distinct from both ATM and XLF.

PAXX promotes the accumulation of $\mathrm{KU}$ at DNA ends in vivo. $\mathrm{KU}$, especially the conserved $\mathrm{N}$-terminal regions required for hetero-dimerization, is conserved through evolution and is essential for DNA end-ligation in vivo and in vitro. PAXX binds to KU directly ${ }^{16,17}$ and is rapidly recruited to DNA damage sites. So we tested whether KU is essential for the recruitment of PAXX to the DSB in vivo. GFP-PAXX was efficiently recruited to the site of laser-induced DNA lesions in $\mathrm{Ku} 80^{+/+}$, but not in $\mathrm{Ku}_{80^{-1}}$ MEFs (Fig. 7d). Moreover both full-length KU80 and a truncated KU80 lacking the flexible C-terminal domain $(\triangle \mathrm{CTD})$ implicated in DNA-PKcs binding, fully restored PAXX recruitment (Fig. $7 \mathrm{~d}$ and Supplementary Fig. 5C), suggesting $\mathrm{PAXX}$ binds to the conserved $\mathrm{N}$-terminal region 
a

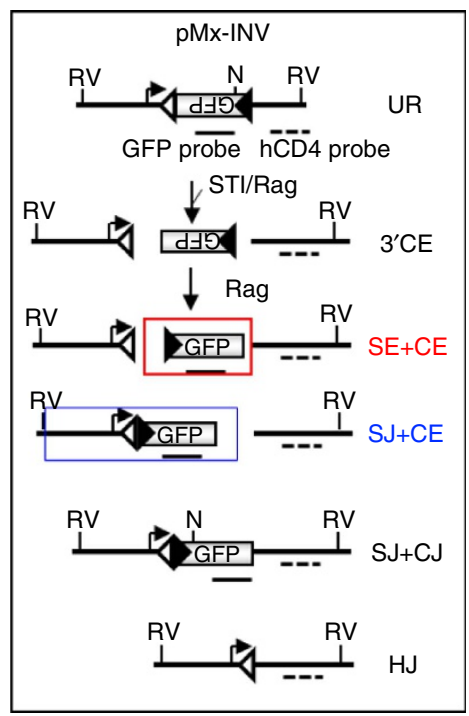

b

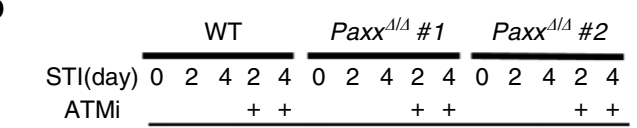

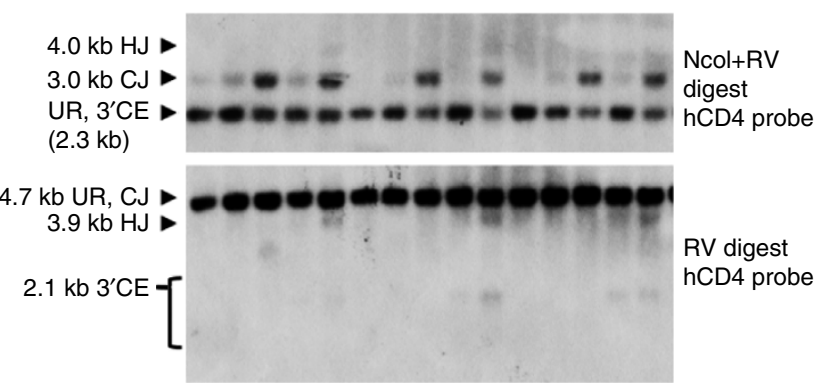

d

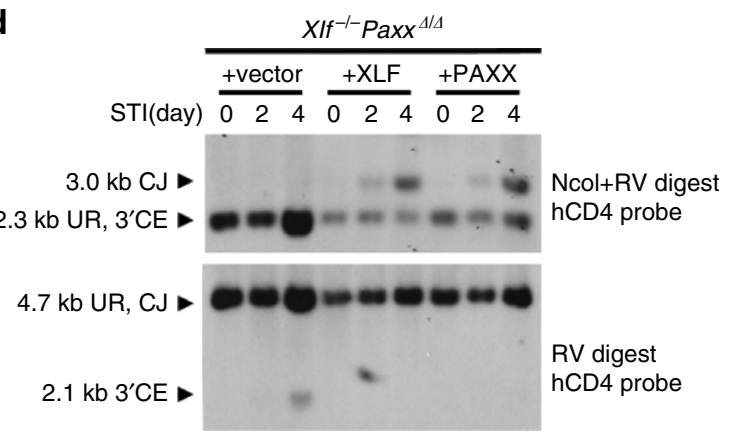

e

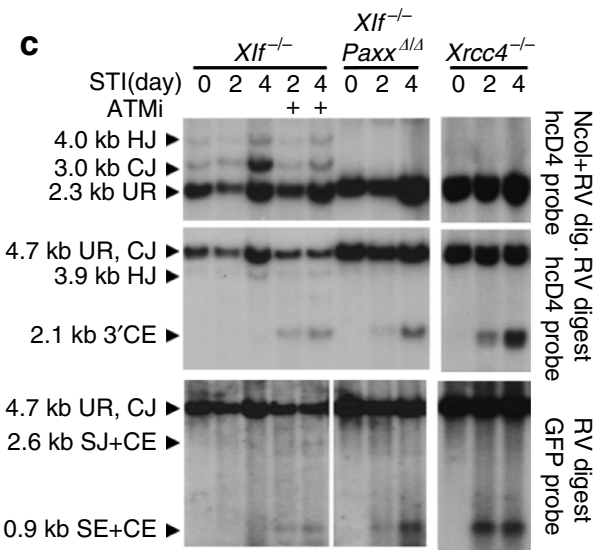

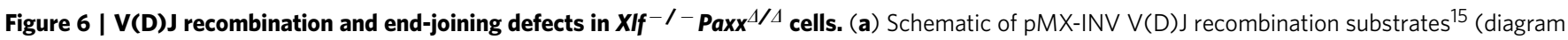
modified from Zha et $a .^{12}$ ). The unrearranged substrate (UR), hybrid joins (HJs), coding/signal end (CE/SE) intermediates and coding/signal joins (CJs/SJs) are diagrammed. The recombination signal sequence (RSS, triangle), GFP probe (solid lines) and hCD4 probe (dash lines) are indicated. Positions of EcoRV (RV) sites and $\mathrm{Ncol}(\mathrm{N})$ sites are shown. The red box shows the isolated SE-CE fragments resulting from complete end-ligation defects. The blue box shows the SJ-CE fragments resulting from isolated CJ formation defects (for example, hairpin opening defects in DNA-PKcS ${ }^{-/-}$cells) ${ }^{3}$.

(b-d) Southern blot analyses of pMX-INV rearrangement products and intermediates. The digestion and probes are marked on the side of the blots. The WT and the two independently derived $\operatorname{Paxx}^{\Delta / \Delta}$ lines in $\mathbf{b}$ have the $\mathrm{pMX}$-INV substrates integrated at the same genomic location. And the XIf- ${ }^{-}$and isogenic $X I f^{-/}-$Paxx $^{\Delta / \Delta}$ derivative have their $\mathrm{PMX}-\mathrm{INV}$ integrated in the same genomic locus. Xrcc $4^{-/}-$cells were included as a control. (e) Diagram of Ppol-mediated cleavage in one mouse genomic DNA site on chromosome 1. H3 = Hind III. The dark line indicates the position of the probe designed to detect DNA ends. Lower: Southern blot analyses for unrepaired ends from representative cells with HindllI-digested DNA.

of KU. Given the importance of $\mathrm{KU}$ in end-ligation, we next asked whether PAXX affects $\mathrm{KU}$ dynamics in vivo and found that loss of PAXX, but not the loss of XLF, significantly reduced the intensity of GFP-KU70 at DSBs in vivo (Fig. 7e). Correspondingly, the intensity of GFP-KU70 also reduced significantly in $\mathrm{Xlf}^{-1-} \mathrm{Paxx}^{-1-} \mathrm{MEFs}$, although consistently higher than in $\mathrm{Paxx}^{-1-}$ cells, likely caused by the continuous recruitment of $\mathrm{KU}$ due to persistent breaks in $\mathrm{Xlf}^{-1-} \mathrm{Paxx}^{-1-}$ cells (Fig. 7e). Meanwhile, the level of GFP-Lig4 is markedly reduced in $\mathrm{Xlf}^{-1-}$ MEFs and nearly absent in $\mathrm{Xlf}^{-1-} \mathrm{Paxx}^{-1-}$ cells (Fig. 7f). At the same time, the recruitment of GFP-Lig4 is at most moderately affected in Paxx ${ }^{-1-}$ cells, which is likely secondary to the KU-recruitment defects (Fig. 7f). Together these data suggest a model in which PAXX promotes robust recruitment of KU, while XLF enhances the recruitment of Lig4, and together they ensure efficient end-ligation through distinct yet complementary mechanisms (Fig. $7 \mathrm{~g}$ ).

\section{Discussion}

PAXX was recently proposed to function as a NHEJ factor on the basis of its structural homology to XRCC4 and XLF, and the IR sensitivity of PAXX-depleted human tumour lines. 
a
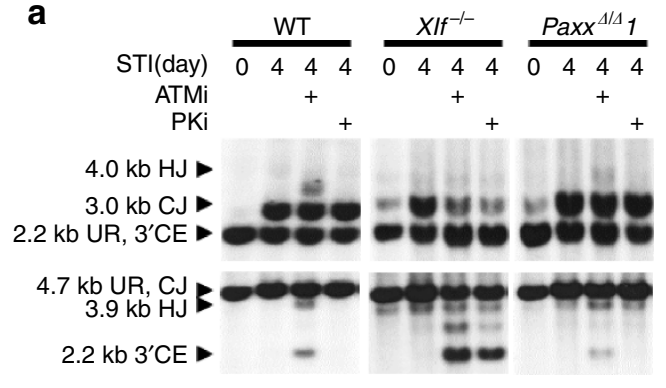

C

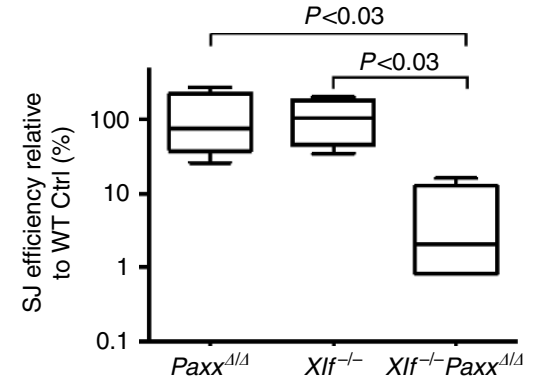

b

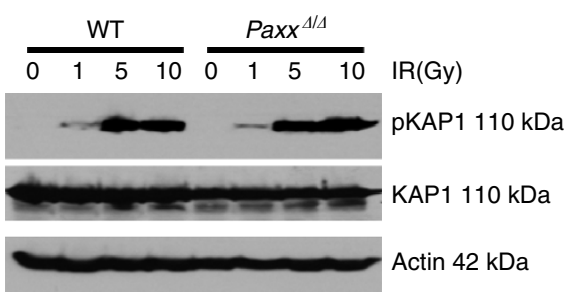

d

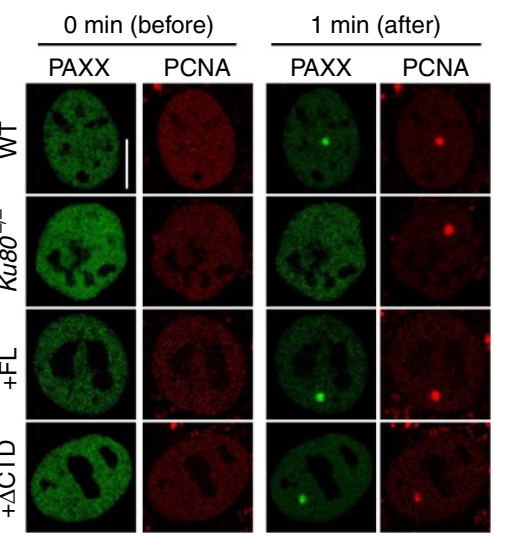

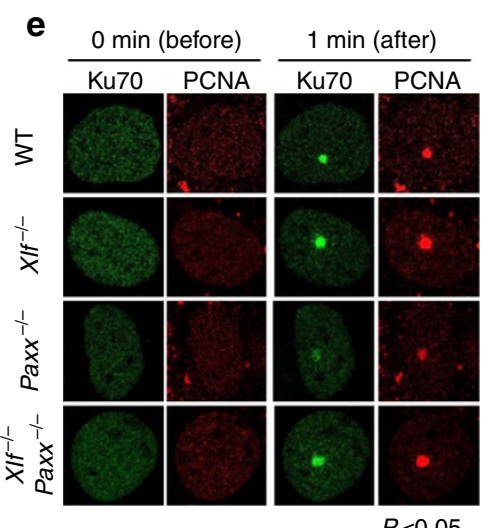

f
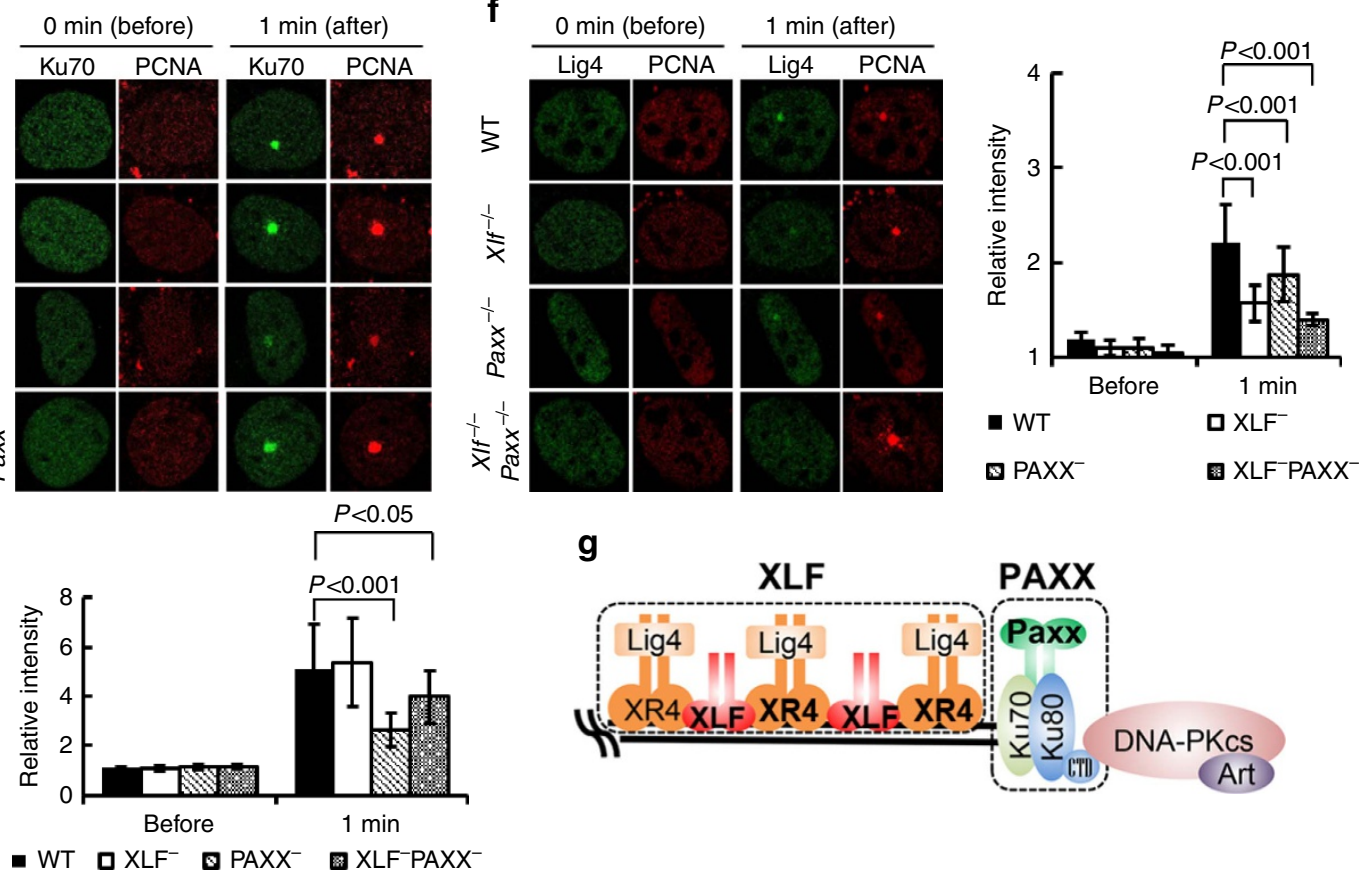

g

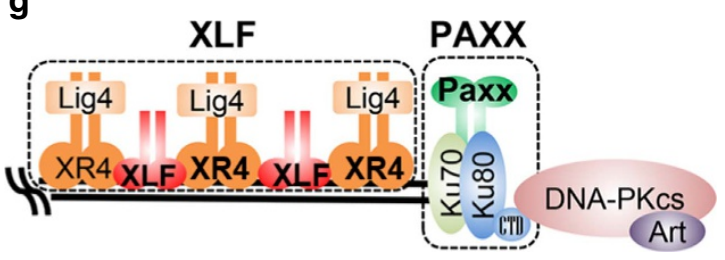

Figure 7 | PAXX and XLF have distinct functions during NHEJ. (a) Southern blot analyses of the product and intermediates of pMX-INV V(D)J recombination reporter in WT, XIf ${ }^{-/-}$and Paxx ${ }^{\Delta / \Delta}$ cells with or without ATM kinase inhibitor (KU55933, $15 \mu \mathrm{M}$ ) or DNA-PKcs inhibitor (NU7441, $5 \mu$ M) added $1 \mathrm{~h}$ before STI571 $(3 \mu \mathrm{M})$. (b) Western blot for phosphorylated KAP1, total KAP1 and $\beta$-actin in total lysate from pre-B cells immediately after irradiation (at 1, 5 or $10 \mathrm{~Gy}$ ). (c) Extrachromosomal SJ formation efficiencies in WT, Paxx ${ }^{\Delta / \Delta}, X^{-} f^{-/}-$and XIf ${ }^{-/}-$Paxx ${ }^{\Delta / \Delta}$ Pre-B cells. Each box represents the average and s.d. of four independent repeats. Box centre line is plotted at the median and the box extends from the 25th to 75th percentiles. Whiskers go down to the smallest value and up to the largest value. The raw data were summarized in Supplementary Fig. 5A. The $P$ value was calculated by twotailed Student's t-test. (d) Laser-induced recruitment of GFP-PAXX or DsRed-PCNA in immortalized Ku80-/ - MEFs with or without ectopic expression of full-length (FL) Ku80 or C-terminal truncated Ku80 ( $\triangle C T D$ ). Ten cells were tested for each genotype and representive images are shown here.

(e) Laser-induced recruitment of GFP-Ku70 in immortalized WT, XIf ${ }^{-/-}$, Paxx ${ }^{-/-}$and $X I f^{-/}-$Paxx ${ }^{-/-}$MEFs 1 min after damage. P<0.001 between Paxx $^{-/-}$and WT cells, $P<0.05$ between $X I f^{-/-}$Paxx ${ }^{-/-}$and WT cells, two-way ANOVA (f) Laser-induced recruitment of GFP-Lig4 in immortalized

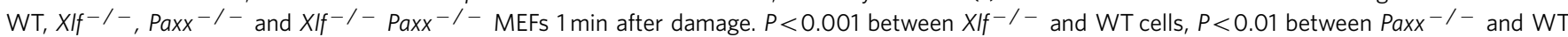
cells, $P<0.001$ between $X I f^{-/-}$Paxx ${ }^{-/-}$and WT cells, two-way ANOVA. The bar graphs of e,f represent the average and error bars stand for s.d. (see Methods for details), same experiments were repeated for at least two times. The scale bar in $\mathbf{d}$ stands for $10 \mu \mathrm{m}$. (g) Working model. In this model, we propose that XLF (diagrammed as red bean sprout shape) enhances the efficient accumulation of Lig4 in complex with XRCC4 at the DNA ends, while PAXX (diagrammed as green bean sprout shape) promotes the accumulation of KU at the DNA ends. Together PAXX and XLF promote efficient DNA end-ligation through distinct mechanisms. XR4, XRCC4; Art, Artemis. 
Unexpectedly, we found that PAXX is largely dispensable for NHEJ in mice under physiological conditions. Lymphocyte development, including both $\mathrm{V}(\mathrm{D}) \mathrm{J}$ recombination and CSR, as well as the general NHEJ repair activity required for maintenance and expansion of peripheral lymphocytes, are not affected by the loss of PAXX. Accordingly, PAXXdeficient primary MEFs proliferated well in culture and did not accumulate significantly more spontaneous chromosomal breaks than WT controls. Yet PAXX-deficient primary MEFs or v-abl kinase-transformed B cells displayed a moderate, but significant, sensitivity to IR. These data indicate that PAXX serves as a genomic caretaker that is largely dispensable under physiological conditions, and instead functions to ensure genomic integrity under stresses or in selective genetic backgrounds. One of these conditions would be XLF deficiency. While neither PAXX nor XLF alone is essential for murine development or lymphocyte $\mathrm{V}(\mathrm{D}) \mathrm{J}$ recombination, $\mathrm{Paxx} \mathrm{I}^{-/-}$mice require $\mathrm{XLF}$ and $X l f^{-1-}$ mice require PAXX for end-ligation. As such, $X l f^{-1-P_{a x x}}-1-$ mice died during late embryonic development with severe neuronal apoptosis similar to $\operatorname{Lig} 4^{-1-}$ or $X r c c 4^{-1-}$ mice ${ }^{10,11}$. Xlf ${ }^{-1-}$ Paxx $^{-1-}$ primary MEFs accumulate high levels of chromosomal breaks, leading to cell cycle arrest, and eventually fail to thrive. $X l f^{-I}-\operatorname{Paxx}^{\Delta / 4}$ lymphocytes cannot complete $\mathrm{V}(\mathrm{D}) \mathrm{J}$ recombination and thus accumulate unrepaired SEs and CEs. Notably, both chromosomal and extra-chromosomal end-joining were abolished in $\mathrm{Xlf}^{-/-}$ $\mathrm{Paxx}^{4 / \Delta}$ cells, supporting a role of PAXX in DNA end-ligation directly. Accordingly, we found that PAXX, but not XLF promotes the maximal recruitment of KU to DNA ends in vivo. Taken together, our findings identified distinct, yet complementary functions of two core NHEJ factors-PAXX and XLF in end-ligation and revealed an intricate regulation of the NHEJ pathway. Consistent with our findings, two recent studies using B-cell lines with CRISPR-Cas9 knockout of PAXX and XLF also revealed their redundant function in end-joining ${ }^{32,33}$.

PAXX shares structural similarity with both XRCC4 and XLF. Unlike XRCC4 (refs 34,35), both PAXX and XLF have relatively short coiled-coil stalks that cannot bind to Lig4 directly and are not absolutely required for end-ligation. In vivo, loss of either XLF or PAXX has negligible effects on the survival of post-mitotic neurons (Fig. 3e), proliferation of primary MEFs (Fig. 4a), or chromosomal $\mathrm{V}(\mathrm{D}) \mathrm{J}$ recombination in developing lymphocytes (Fig. 6). While these phenotypes are strikingly similar, there are notable differences. Loss of XLF reduced the cellularity of peripheral lymphoid organs and caused a $\sim 50 \%$ reduction in $\mathrm{CSR}^{30}$, while PAXX deficiency has no measurable impact on CSR or the cellularity and growth of lymphoid organs (Fig. 2) ${ }^{32}$. In addition, XLF-deficient MEFs exhibit spontaneous genomic instability, while PAXX-deficiency did not lead to statistically significant increases in genomic instability (Fig. 4). Moreover, PAXX-deficient MEFs exhibit little, if any, sensitivity to IR, in contrast to the moderate IR sensitivity of XLF-deficient cells (Figs 4 and 5). Most strikingly, ATM and DNA-PKcs kinase activities are required for chromosomal end-joining in XLFdeficient cells, but are dispensable in PAXX-deficient cells (Fig. 7). At the molecular level, XLF forms filamental structures with XRCC4, which promotes Lig4 re-adenylation in vitro ${ }^{36}$. Here we found that XLF also promotes efficient recruitment of Lig4 in vivo without affecting $\mathrm{KU}$ (Fig. 7f). In contrast, PAXX binds directly to DNA-bound KU and promotes accumulation of KU at DSBs in a reciprocal manner (Fig. 7e). Based on these data, we proposed that while XLF and PAXX have important complementary activities, they function in distinct protein complexes, and through different mechanisms to ensure end-ligation (Fig. 7g).
So what is the role of PAXX in end-joining? Like DNA-PKcs, PAXX interacts with DNA bound KU, but not free KU. But in contrast to DNA-PKcs, which interacts with the C-terminal tails of $\mathrm{Ku} 80$, PAXX requires the core of $\mathrm{KU}$, but not the Ku80 C-terminal domain, for its recruitment to DNA damage sites (Fig. 7). We found that in the absence of PAXX, the maximum intensity of GFP-Ku70 at the DNA damage site reduced significantly (Fig. 7). Given KU is essential for the initial recruitment of PAXX, we postulate that PAXX might stabilize KU at the DNA ends, rather than affecting the initial recruitment of KU to DNA. Since $K u$ deletion rescues the embryonic lethality of Lig4-deficient mice ${ }^{37}$, presumably by allowing the alternative end-joining (A-EJ) pathway to access the DNA ends, this role of PAXX in stabilizing KU might explain why knockout of Paxx in Xrcc4 $4^{-/-}$chicken DT40 cells partially rescues the severe IR sensitivity ${ }^{17}$. If so, why do Xlf ${ }^{-1-}$ Paxx $^{-1-}$ embryos die of severe neuronal apoptosis? We note that the intensity of Lig4 foci is lowest in $X l f^{-/-}$Paxx $^{-/}$cells, while the intensity of $\mathrm{Ku}$ foci is actually higher in $X l f^{-I-} \mathrm{Paxx}^{-I}$ cells than in Paxx-1- cells (Fig. 7e,f), likely due to the continuous recruitment of $\mathrm{KU}$ in the absence of DNA repair. This recruitment pattern in $X l f^{-/-} \mathrm{Paxx}^{-/-}$cells (the presence of KU without Lig4) is similar to that found in the Lig4 ${ }^{-1-}$ cells, where both NHEJ (due to Lig4 deficiency) and A-EJ (due to the presence of $\mathrm{KU}$ ) are limited, and might explain the severe endligation defects and embryonic lethality of $X^{-1-} f^{-1} x^{-1-}$ mice. In this context, the sensitivity to genotoxic agents or spontaneous damages would be determined by a balance and availability of NHEJ and A-EJ to given types of breaks. Given the robust NHEJ in Paxx ${ }^{-1-}$ mice, PAXX-dependent stabilization of KU at DNA ends is clearly not essential for NHEJ in otherwise WT cells. This function of PAXX becomes critical when XLF is also absent in the cells. XLF-deficient cells also require ATM, DNA-PKcs, 53BP1 and H2AX for end ligation ${ }^{5,12-14,24,28}$. In addition, the $\mathrm{C}$ terminus of the RAG2 protein is required for chromosomal $\mathrm{V}(\mathrm{D}) \mathrm{J}$ recombination specifically in XLF-deficient cells $^{28}$. ATM and its substrates are thought to increase DNA end stability while the $\mathrm{C}$ terminus of RAG has been proposed to facilitate end-ligation in XLF-deficient cells during $V(D) J$ recombination by stabilizing the post-cleavage complex embracing the DNA ends ${ }^{15,38}$. Therefore, it is conceivable that PAXX might also facilitate end-ligation by promoting end stability and synapsis similar to RAG2 during $\mathrm{V}(\mathrm{D}) \mathrm{J}$ recombination and to ATM/DNA-PK through their chromatinbound substrates. Consistent with their similar function, end-ligation in the PAXX-deficient cells is not further affected by loss of ATM or DNA-PK kinase activity or in RAG-independent breaks (Figs 6e and $7 \mathrm{a})^{32,33}$. KU has been proposed to have synaptic function through the recruitment and intermolecular phosphorylation of DNA-PKcs ${ }^{39,40}$. Given the direct interaction between PAXX and KU and the role of PAXX in the accumulation of KU at DSBs in vivo (Fig. $7 \mathrm{~d}, \mathrm{e}$ ), it is tempting to speculate that PAXX modulates the stability of the DNA-PKcs/KU-DNA complex to promote end-synapsis and support end-ligation in XLF-deficient cells. Consistent with this model, inhibiting DNA-PK kinase activity has no additional impact on end-ligation in Paxx ${ }^{-1-}$ cells (Fig. 7a).

PAXX is the first bona fide NHEJ factor that was not firstly identified in patients or as naturally occurring mutations in another organism. Patients with loss-of-function mutations of Lig4, XRCC4, XLF, Artemis and DNA-PKcs develop SCID and variable levels of microcephaly ${ }^{1,2}$. Given the severe IR sensitivity observed in PAXX-depleted human tumour lines, patients with PAXX deficiencies have been expected. Yet, the mild phenotype of Paxx $^{-1-}$ mice suggests that PAXX deficiency might not be readily recognized. While the precise reason for the 
strong dependence on PAXX in NHEJ in human cancer cell lines and the dispensable role of PAXX in mice in vivo is yet to be determined, species differences are unlikely to be the only explanation. While in U2OS cells and $293 \mathrm{H}$ cells, PAXX deficiency causes severe IR sensitivity, loss of PAXX causes only moderate, if any, IR sensitivity in human colorectal cancer HCT116 cells and 293 cells ${ }^{17,24}$. It is possible that genetic heterogeneity, including the availability of other repair factors might contribute to this variability. So what would be the physiologic function of the evolutionarily conserved PAXX gene? One possibility is that PAXX may promote tumour suppression or prevent premature aging in long-living organisms. While lymphocyte numbers are only moderately reduced in young XLF-deficient mice, old XLF-deficient mice (24 mos.) display rapid reduction of lymphocyte numbers owing to decreased renewal and function of the hematopoietic cells ${ }^{41}$. If a similar phenotype is observed in older $\operatorname{Paxx}^{-7-}$ mice, then PAXX-deficient patients, if they exist, might present premature ageing phenotypes such as late-onset immunodeficiency or myeloid proliferative diseases. Except rare truncating mutations in the C-terminal KU-binding region of PAXX, the PAXX (C9Orf142) gene is amplified and overexpressed in several human cancers (cBioPortal), suggesting that PAXX function might modulate the therapeutic responses to genotoxic cancer therapies, such as radiation.

\section{Methods}

Mice. To generate $\mathrm{Paxx}-1-$ mice, a targeting construct was made to replace part of Exon 1 (after ATG) and exons 2-7 of the Paxx gene with a neomycin resistance gene. To generate the Paxx targeting construct, $5^{\prime}(\sim 2 \mathrm{~kb})$ and $3^{\prime}$ (3.4 kb, with HindIII linker) arms were PCR amplified from $129 / \mathrm{Sv}$ murine ES cell DNA with high fidelity DNA polymerase (Phusion, NEB), cloned into pGEMT shuttle vectors (Promega) and sequenced (Genewiz). The primers used for the PCR are listed in Supplementary Table 2. The $5^{\prime}$ arm and $3^{\prime}$ arm were then released and subcloned to the pEMC targeting vector with NeoR flanked by frt sites using HindIII or NotI respectively. The final vector was validated by sequencing and restriction digestions. ClaI-linearized pEMC-PAXX plasmid was electroporated into CSL3 murine ES cells (129/Sv) and selected with G418 $\left(300 \mu \mathrm{g} \mathrm{ml}^{-1}\right)$ and Ganciclovir (Sigma G2566, $2 \mu \mathrm{M}$ ) for 7 days. The correct targeted clones were identified by Southern blotting analyses using EcoRV-digested genomic DNA and a $3^{\prime}$ genomic probe as outlined in Fig. 1a (the primers used to generate the probe are listed in Supplementary Table.2). The WT band is $\sim 4.7 \mathrm{~kb}$, and the targeting destroys one of the EcoRV site in Exon 3, resulting in a $\sim 7.0 \mathrm{~kb}$ band. A total of eight independent targeted ES cells were obtained and two were injected for germline transmission and yielded identical phenotypes. Genotyping was performed with primers listed in Supplementary Table 2. Primers used to generate the mPAXX probes are also listed in Supplementary Table 2. Xlf ${ }^{-1-}$ mice ${ }^{7,42}$ were bred to $\mathrm{Paxx}^{-1-}$ mice to generate $X l f^{-1-} \mathrm{Paxx}^{-1-}$ mice and $53 B P 1^{-1-}$ mice $^{43}$ (Supplementary Fig. 1D) were used as a control for thymocyte development defects. Both male and female mice were used in all experiments. All animal work has been conducted in a specific pathogen-free facility and all the procedures were approved by Institutional Animal Care and Use Committee (IACUC) at Columbia University Medical Center.

Lymphocyte development and recombination. Single-cell suspensions were prepared from lymphocytes of thymus (Thy), bone marrow (BM), spleen (Spl) and lymph node (LN) from 7-week-old mice of the described genotypes and $\sim 1 \times 10^{5}$ cells were stained using fluorescence-conjugated antibodies as indicated before analysed by flow cytometry. For Class Switch Recombination assay, single-cell suspensions of spleen cells were sorted with CD43 magnetic beads (MACS, Miltenyi), and B cells were cultured at a density of $5 \times 10^{5}$ cells per $\mathrm{ml}$ in RPMI medium supplemented with $10 \%$ FBS and $25 \mathrm{ng} \mathrm{ml}^{-1}$ of LPS plus $25 \mathrm{ng} \mathrm{ml}^{-1}$ of IL-4 (R\&D). Cultured cells were maintained daily at a density of $1 \times 10^{6}$ cells per ml. Cells were collected on various days for flow cytometry. Singlecell suspensions from spleens were prepared according to the standard methods from mice 6 to 12 weeks old. Cells from cultures on day 4 were washed twice in PBS plus $2 \%$ FBS and were stained with various antibodies conjugated with fluorescein isothiocyanate (IgG1, BD Pharmingen and B220, eBioscience). Flow cytometry was performed on a FACS Calibur flow cytometer (BD Bioscience) and data were processed using FlowJo software package.

Endogenous Vbeta14DJbeta1 junction analyses. Vbeta14DJbeta1 coding joins were amplified from total thymocyte DNA with the following primers: Vbeta14F:
5'-AGAGTCGGTGGTGCAACTGAACCT-3' and Jb1.2 R: 5'-CCTGACTT CCACCCGAGGTTC- $3^{\prime}$. Two amplicons were identified from each sample corresponding to rearrangement involving J $\beta 1.1$ or J $\beta 1.2$. The two amplicons were gel-purified separately and cloned into a pGEM-T Easy Vector (Promega) following the manufacturer's protocol. Individual clones were sequenced with the built-in T7 primer site and aligned with the V $\beta 14$ and J $\beta$ sequence (accession no. AE000665). Sequence analyses identified the V, D, J coding region first, then identified Palindromic (P) element and non-template nucleotide addition $(\mathrm{N})$. The number of nucleotide deletions for the V-D junction and D-J junctions were also calculated (Supplementary Table.1).

Histological analyses. Embryo heads were fixed in $4 \%$ paraformaldehyde, paraffin embedded, serially sectioned (sagittal, $5 \mu \mathrm{m}$ ) and stained with hematoxylin and eosin (H\&E) or cleaved Caspase-3 (Cat\#9664, Cell Signaling) in the standard histopathology core of Columbia University.

Proliferation and chemosensitivity. Primary MEFs isolated from E14.5 embryos were designated passage 0 (P0) and were cultured in DMEM medium (GIBCO, Life Technology) containing 15\% fetal bovine serum, $100 \mathrm{mM}$ L-glutamine, and penicillin-streptomycin on a gelatinized plate until they reached confluence. Equal numbers of cells $\left(1 \times 10^{4}\right)$ were plated into a well of a six-well plate. The medium was changed daily and MEFs were counted at different time points in triplicate using a hemocytometer.

For IR, etoposide and HU sensitivity assays, cells were plated on 96-well plates $24 \mathrm{~h}$ before irradiation or the addition of genotoxic agents (etoposide: $0.01,0.1$ and $1 \mu \mathrm{M}$; hydroxyurea: 20,200 and 2,000 mM. The drug was washed away $24 \mathrm{~h}$ after the treatment). The relative cell density was determined 7 days after irradiation or drug treatment by fluorescence nucleotide dye CyQuant (MEFs) or by hemocytometer ( $\mathrm{v}$-abl transformed pre-B cells).

For cell cycle analyses, proliferating primary MEF cells were incorporated with $10 \mu \mathrm{M}$ BrdU for $30 \mathrm{~min}$ and fixed and then stained with Anti-BrdU FITC (Cat\# 11-5071-41, eBioscience) and propidium iodide (PI) and analysed by flow cytometry

Metaphase preparation and telomere-FISH staining. The metaphase was prepared as previously described ${ }^{3}$. Briefly, cells were cultured for 6 (primary MEF) or 3 (v-abl transformed pre-B cells) hours with colcemid (KaryoMAX Colcemid Solution, GIBCO) to the final concentration of $100 \mathrm{ng} \mathrm{ml}^{-1}$. The treated cells were then collected and swollen using hypotonic solution at room temperature and fixed (fresh fixative made by 3:1 v/v methanol: acetic acid). Metaphase spreads were obtained by dropping fixed cells onto pre-cleaned slides. Slides were fixed in $4 \%(\mathrm{w} / \mathrm{v})$ formaldehyde/PBS, followed by three washes with PBS and digestion with pepsin/PBS $(0.1 \%$; Sigma). The slides were then washed three times in PBS and dehydrated through ethanol, serially. A Cy3-labelled (CCCTAA) 3 peptide nucleic acid (PNA) probe (customer synthesized, Biosynthesis Inc.) was used to hybridize the metaphases under denaturing conditions (heating for $3 \mathrm{~min}$ at $80^{\circ} \mathrm{C}$ on a hea block) and incubated in a dark humidity chamber for $2 \mathrm{~h}$. The slides were washed and dehydrated in ethanol and air dried. DNA was counterstained with DAPI. A minimum of 100 cells with telomere signals were captured by Metafer 4 using Metasystems automatic Metaphase search and scan system equipped with a Plan Fluor Nikon Lens $(\times 63 / 1.30$ Oil, Japan) and counted for breaks.

Cas9-mediated deletion of Paxx. A pair of guide RNAs (gRNA) targeting the mouse Paxx gene were designed and cloned into the pX330 CRISPR plasmid (generously provided by Dr Feng Zhang through Addgene) (gRNA1: $5^{\prime}$-CACC GCTAAGGTGTTCGCTCGGCGG-3'; gRNA2: 5'-CACCGCAGTTTATTTGACG GAGAA- $3^{\prime}$ ). The pair of gRNA containing plasmids were electroporated into $\mathrm{v}$-abl kinase-transformed WT or Xlf $f^{-/} \mathrm{v}$-abl transformed pre-B cells using the 4D Nucleofector apparatus (Buffer SF, program DN100, Lonza, Walkersville, MD). The cells were plated for single clones, and PCR (5'-ATTGAAGAGCGGCAGA TATGT- $3^{\prime}$ and $5^{\prime}$-ACGCAGAATCAACACAGTAGGT- $3^{\prime}$ ) was performed on each single clone to identify the deletion. When necessary, heterozygous targeted clones were targeted a second time to remove the second allele. Complete deletion was verified by PCR, Southern blot and western blot.

Construction of plasmids. pBMN-IRES-GFP was purchased from Addgene and the GFP was replaced by a truncated humanCD2 fragment to generate pBMNIRES-hCD2, a Flag sequence was then inserted in front of the multiple cloning site of pBMN-IRES-hCD2 to generate pBMNFlag-IRES-hCD2. Full-length mouse PAXX was inserted into the EcoRI digested pBMNFlag-IRES-hCD2. pBMN-XLFIRES-hCD2 and pBMN-FLAG-KU80-IRES-hCD2 and pBMN-FLAGKU80 $\Delta$ CTD-IRES-hCD2 were described previously ${ }^{3,13}$.

Western blot and antibodies. Cell or tissue lysates were prepared in RIPA buffer ( $50 \mathrm{mM}$ Tris- $\mathrm{HCl} \mathrm{pH} 8.0,150 \mathrm{mM} \mathrm{NaCl}, 0.1 \%$ SDS and $0.5 \%$ sodium deoxycholate $1 \%$ NP40 and fresh proteinase inhibitor cocktail). Protein extracts were analysed by western blotting according to the standard protocols using primary antibodies 
specific for PAXX (ab126353, Abcam, 1:1,000 dilution), XLF (A300-730A-1, Bethyl Laboratories, 1:1,000 dilution), Flag (M2, Sigma-Aldrich, 1:10,000 dilution), KAP1 (ab10484, Abcam, 1:1,000 dilution), phospho-KAP1 (ab70369, Abcam, 1:1,000 dilution). HRP-conjugated anti-rabbit and mouse secondary antibodies (GE Healthcare) were used and signal was detected using an ECL western blotting detection system (GE Healthcare). Uncropped images of all blots are available in Supplementary Fig. 6.

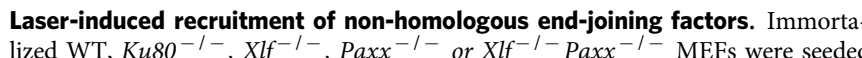
onto glass bottom $35 \mathrm{~mm}$ diameter plates at about $10^{4}$ cells per plate. Transient transfection of $1 \mu \mathrm{g}$ GFP-Ku70 or $1 \mu \mathrm{g}$ GFP-PAXX or $1 \mu \mathrm{g}$ GFP-Lig4, respectively, with $1 \mu \mathrm{g}$ DsRed-PCNA were conducted using Lipofectamine 2000 (ThermoFisher) following the manufacturer's instruction. To obtain reliable recruitment of GFP-Lig4 with the $405 \mathrm{~nm}$ laser, the transfected cells were sensitized with $10 \mu \mathrm{M}$ of BrdU overnight before imaging. Live cell imaging combining laser micro-irradiation with confocal microscope was carried out using Nikon Ti Eclipse inverted microscope (Nikon, Inc.) equipped with A1 RMP (Nikon, Inc.) confocal microscope system (Nikon, Inc.) and Lu-N3 Laser Units (Nikon, Inc.). Laser micro-irradiation manipulation and time-lapse imaging was performed with the NIS Element High Content Analysis software (Nikon, Inc.), using a $405 \mathrm{~nm}$ laser with $100 \%$ energy level (output power on sample $\cong 110 \mu \mathrm{W}$, adequate for Ku70 accumulation). Relative intensity at laser-damaged sites was calculated as the mean value of the ratio of intensity of each micro-irradiation damaged sites to whole nucleus background. More than 10 individual cells were randomly chosen, tested and analysed for each data point. Mean value, standard deviation and outliers were calculated with Excel and GraphPad Prism online tools. For GFPKu70 recruitment, WT shows the average of 9 cells, $X^{-1} f^{-1}$ shows the average of 12 cells, $\mathrm{Paxx}^{-1-}$ shows the average of 21 cells and $\mathrm{Xlf}^{-1-} \mathrm{Paxx}^{-1-}$ shows the average of 12 cells. For GFP-Lig4 recruitment, WT shows the average of 10 cells, $X^{-1-}$ shows the average of 8 cells, Paxx ${ }^{-I-}$ shows the average of 11 cells and $\mathrm{Xlf}^{-1-} \mathrm{Paxx}^{-1-}$ shows the average of 10 cells. In both GFP-Ku70 and GFP-lig4 recruitment, results of $\mathrm{Xlf}^{-I^{-}}, \mathrm{Paxx}^{-I_{-}^{-}}$and $\mathrm{Xlf}^{-I_{-}^{-}} \mathrm{Paxx^{-l_{- } }}$ MEFs were compared with WT with two-way ANOVA to calculate the $P$ value.

Transient V(D)J recombination assay. Extra-chromosomal V(D)J recombination assays were performed in $\mathrm{v}$-abl transformed cell lines by transfection with JH200 (signal join) substrate plasmids as previously described. ${ }^{3} \mathrm{~V}(\mathrm{D}) \mathrm{J}$ recombination efficiency was determined by the number of chloramphenicolampicillin double-resistant bacterial colonies among the total ampicillin-resistant colonies. The relative efficiency of SJ formation was calculated by setting the chloramphenicol-ampicillin double-resistant/total ampicillin-resistant colonies in the WT cells in each biological replicate as $100 \%$.

Data availability. V $\beta 14$ and $\mathrm{J} \beta$ sequence is available from the NCBI under Accession No. AE000665. The authors declare that all data supporting the findings of this study are available from the corresponding author upon request.

\section{References}

1. Lieber, M. R. The mechanism of double-strand DNA break repair by the nonhomologous DNA end-joining pathway. Annu. Rev. Biochem. 79, 181-211 (2010).

2. Woodbine, L., Gennery, A. R. \& Jeggo, P. A. The clinical impact of deficiency in DNA non-homologous end-joining. DNA Repair 16, 84-96 (2014).

3. Jiang, W. et al. Differential phosphorylation of DNA-PKcs regulates the interplay between end-processing and end-ligation during nonhomologous end-joining. Mol. Cell 58, 172-185 (2015).

4. Bassing, C. H., Swat, W. \& Alt, F. W. The mechanism and regulation of chromosomal V(D)J recombination. Cell 109 (Suppl) S45-S55 (2002).

5. Lescale, C. et al. RAG2 and XLF/Cernunnos interplay reveals a novel role for the RAG complex in DNA repair. Nat. Commun. 7, 10529 (2016).

6. Franco, S., Alt, F. W. \& Manis, J. P. Pathways that suppress programmed DNA breaks from progressing to chromosomal breaks and translocations. DNA Repair 5, 1030-1041 (2006).

7. Li, G. et al. Lymphocyte-specific compensation for XLF/cernunnos end-joining functions in V(D)J recombination. Mol. Cell 31, 631-640 (2008).

8. Boboila, C. et al. Alternative end-joining catalyzes class switch recombination in the absence of both Ku70 and DNA ligase 4. J. Exp. Med. 207, 417-427 (2010).

9. Frank, K. M. et al. Late embryonic lethality and impaired V(D)J recombination in mice lacking DNA ligase IV. Nature 396, 173-177 (1998).

10. Frank, K. M. et al. DNA ligase IV deficiency in mice leads to defective neurogenesis and embryonic lethality via the p53 pathway. Mol. Cell 5, 993-1002 (2000).

11. Gao, Y. et al. A critical role for DNA end-joining proteins in both lymphogenesis and neurogenesis. Cell 95, 891-902 (1998).

12. Zha, S. et al. ATM damage response and XLF repair factor are functionally redundant in joining DNA breaks. Nature 469, 250-254 (2011).
13. Liu, X. et al. Overlapping functions between XLF repair protein and 53BP1 DNA damage response factor in end joining and lymphocyte development. Proc. Natl Acad. Sci. USA 109, 3903-3908 (2012).

14. Oksenych, V. et al. Functional redundancy between repair factor XLF and damage response mediator 53BP1 in $\mathrm{V}(\mathrm{D}) \mathrm{J}$ recombination and DNA repair. Proc. Natl Acad. Sci. USA 109, 2455-2460 (2012).

15. Bredemeyer, A. L. et al. ATM stabilizes DNA double-strand-break complexes during V(D)J recombination. Nature 442, 466-470 (2006).

16. Ochi, T. et al. DNA repair. PAXX, a paralog of XRCC4 and XLF, interacts with $\mathrm{Ku}$ to promote DNA double-strand break repair. Science 347, 185-188 (2015)

17. Xing, M. et al. Interactome analysis identifies a new paralogue of XRCC4 in non-homologous end joining DNA repair pathway. Nat. Commun. 6, 6233 (2015).

18. Craxton, A. et al. XLS (c9orf142) is a new component of mammalian DNA double-stranded break repair. Cell Death Differ. 22, 890-897 (2015).

19. Li, Y. et al. Crystal structure of human XLF/Cernunnos reveals unexpected differences from XRCC4 with implications for NHEJ. EMBO J. 27, 290-300 (2008).

20. Andres, S. N., Modesti, M., Tsai, C. J., Chu, G. \& Junop, M. S. Crystal structure of human XLF: a twist in nonhomologous DNA end-joining. Mol. Cell 28, 1093-1101 (2007).

21. Ahnesorg, P., Smith, P. \& Jackson, S. P. XLF interacts with the XRCC4-DNA ligase IV complex to promote DNA nonhomologous end-joining. Cell 124, 301-313 (2006).

22. Buck, D. et al. Cernunnos, a novel nonhomologous end-joining factor, is mutated in human immunodeficiency with microcephaly. Cell 124, 287-299 (2006).

23. Ropars, V. et al. Structural characterization of filaments formed by human Xrcc4-Cernunnos/XLF complex involved in nonhomologous DNA end-joining. Proc. Natl Acad. Sci. USA 108, 12663-12668 (2011).

24. Roy, S. et al. XRCC4/XLF interaction is variably required for DNA repair and is not required for ligase IV stimulation. Mol. Cell. Biol. 35, 3017-3028 (2015).

25. Farley, F. W., Soriano, P., Steffen, L. S. \& Dymecki, S. M. Widespread recombinase expression using FLPeR (flipper) mice. Genesis 28, 106-110 (2000).

26. Borghesani, P. R. et al. Abnormal development of Purkinje cells and lymphocytes in Atm mutant mice. Proc. Natl Acad. Sci. USA 97, 3336-3341 (2000).

27. Difilippantonio, S. et al. 53BP1 facilitates long-range DNA end-joining during V(D)J recombination. Nature 456, 529-533 (2008).

28. Oksenych, V. et al. Functional redundancy between the XLF and DNA-PKcs DNA repair factors in V(D)J recombination and nonhomologous DNA end joining. Proc. Natl Acad. Sci. USA 110, 2234-2239 (2013).

29. Orii, K. E., Lee, Y., Kondo, N. \& McKinnon, P. J. Selective utilization of nonhomologous end-joining and homologous recombination DNA repair pathways during nervous system development. Proc. Natl Acad. Sci. USA 103, 10017-10022 (2006).

30. Li, G. et al. Lymphocyte-specific compensation for XLF/Cernunnos end-joining functions in V(D)J recombination. Mol. Cell 31, 631-640 (2008).

31. Franco, S. et al. H2AX prevents DNA breaks from progressing to chromosome breaks and translocations. Mol. Cell 21, 201-214 (2006).

32. Kumar, V., Alt, F. W. \& Frock, R. L. PAXX and XLF DNA repair factors are functionally redundant in joining DNA breaks in a G1-arrested progenitor B-cell line. Proc. Natl Acad. Sci. USA 113, 10619-10624 (2016).

33. Lescale, C. et al. Specific roles of XRCC4 paralogs PAXX and XLF during V(D)J recombination. Cell Rep. 16, 2967-2979 (2016).

34. Sibanda, B. L. et al. Crystal structure of an Xrcc4-DNA ligase IV complex. Nat. Struct. Biol. 8, 1015-1019 (2001).

35. Bryans, M., Valenzano, M. C. \& Stamato, T. D. Absence of DNA ligase IV protein in XR-1 cells: evidence for stabilization by XRCC4. Mutat. Res. 433, 53-58 (1999).

36. Riballo, E. et al. XLF-Cernunnos promotes DNA ligase IVXRCC4 readenylation following ligation. Nucleic Acids Res. 37, 482-492 (2009).

37. Karanjawala, Z. E. et al. The embryonic lethality in DNA ligase IV-deficient mice is rescued by deletion of $\mathrm{Ku}$ : implications for unifying the heterogeneous phenotypes of NHEJ mutants. DNA Repair 1, 1017-1026 (2002).

38. Deriano, L. et al. The RAG2 C terminus suppresses genomic instability and lymphomagenesis. Nature 471, 119-123 (2011).

39. Douglas, P. et al. Polo-like kinase 1 (PLK1) and protein phosphatase 6 (PP6) regulate DNA-dependent protein kinase catalytic subunit (DNA-PKcs) phosphorylation in mitosis. Biosci. Rep. 34, e00113 (2014).

40. Graham, T. G., Walter, J. C. \& Loparo, J. J. Two-stage synapsis of DNA ends during non-homologous end joining. Mol. Cell 61, 850-858 (2016).

41. Avagyan, S. et al. Hematopoietic stem cell dysfunction underlies the progressive lymphocytopenia in XLF/Cernunnos deficiency. Blood 124, 1622-1625 (2014)

42. Zha, S., Alt, F. W., Cheng, H. L., Brush, J. W. \& Li, G. Defective DNA repair and increased genomic instability in Cernunnos-XLF-deficient murine ES cells. Proc. Natl Acad. Sci. USA 104, 4518-4523 (2007) 
43. Ward, I. M., Minn, K., van, D. J. \& Chen, J. p53 binding protein 53BP1 is required for DNA damage responses and tumor suppression in mice. Mol. Cell Biol. 23, 2556-2563 (2003).

\section{Acknowledgements}

We thank Dr Richard Baer for his comments and critical review of the manuscript. We thank Dr Andre Nussenzweig for providing the Ku80-deficient mice, from which we derived Ku80-deficient MEFs. We also thank Drs. Xiaochun Yu, Li Lan and David J. Chen for providing plasmids encoding tagged NHEJ factors used for live cell imaging. We also wish to thank Chen Li and Dr Chyuan-Sheng Lin for exceptional technical assistances in creating genetically modified mouse models. We apologize to colleagues, whose work could not be cited due to space limitations and was covered by reviews instead. This work is in part supported by NIH 5R01CA158073, 5R01CA184187, 1P01CA174653 and American Cancer Society Research Scholar Grant (RSG-13-038-01 DMC) to S.Z. S.Z. is the recipient of the Leukemia and Lymphoma Society Scholar Award. X.L. is a recipient of the Leukemia and Lymphoma Society Career Development Program Fellowship Award. W.J. was supported by NIH/NCI T32 training grant on Cancer Biology (NIH/NCI 5T32CA09503).

\section{Author contributions}

X.L., Z.S., W.J. and B.J.L. conducted the experiments. X.L., Z.S. and S.Z. designed the experiments and analysed the data. X.L., Z.S. and S.Z. wrote the manuscript.

\section{Additional information}

Supplementary Information accompanies this paper at http://www.nature.com/ naturecommunications

Competing financial interests: The authors declare no competing financial interests.

Reprints and permission information is available online at http://npg.nature.com/ reprintsandpermissions/

How to cite this article: Liu, X. et al. PAXX promotes KU accumulation at DNA breaks and is essential for end-joining in XLF-deficient mice. Nat. Commun. 8, 13816 doi: $10.1038 /$ ncomms13816 (2017).

Publisher's note: Springer Nature remains neutral with regard to jurisdictional claims in published maps and institutional affiliations.

(c) (i) This work is licensed under a Creative Commons Attribution 4.0 International License. The images or other third party material in this article are included in the article's Creative Commons license, unless indicated otherwise in the credit line; if the material is not included under the Creative Commons license, users will need to obtain permission from the license holder to reproduce the material. To view a copy of this license, visit http://creativecommons.org/licenses/by/4.0/

(C) The Author(s) 2017 